\title{
The history of X-ray free-electron lasers
}

\author{
C. Pellegrini ${ }^{1,2, \text { a }}$ \\ 1 University of California at Los Angeles, Los Angeles, 90095-1547 California, USA \\ 2 SLAC National Accelerator Laboratory, Menlo Park, 94025 California, USA
}

Received 7 December 2011 / Received in final form 16 March 2012

Published online (Inserted Later)

(C) EDP Sciences, Springer-Verlag 2012

\begin{abstract}
The successful lasing at the SLAC National Accelerator Laboratory of the Linear Coherent Light Source (LCLS), the first X-ray free-electron laser (X-ray FEL), in the wavelength range 1.5 to $15 \AA$, pulse duration of 60 to few femtoseconds, number of coherent photons per pulse from $10^{13}$ to $10^{11}$, is a landmark event in the development of coherent electromagnetic radiation sources. Until now electrons traversing an undulator magnet in a synchrotron radiation storage ring provided the best X-ray sources. The LCLS has set a new standard, with a peak X-ray brightness higher by ten orders of magnitudes and pulse duration shorter by three orders of magnitudes. LCLS opens a new window in the exploration of matter at the atomic and molecular scales of length and time. Taking a motion picture of chemical processes in a few femtoseconds or less, unraveling the structure and dynamics of complex molecular systems, like proteins, are some of the exciting experiments made possible by LCLS and the other X-ray FELs now being built in Europe and Asia. In this paper, we describe the history of the many theoretical, experimental and technological discoveries and innovations, starting from the 1960s and 1970s, leading to the development of LCLS.
\end{abstract}

\section{A new science}

From their discovery by Roentgen in 1895 [Roentgen 1995] X-rays have been one of the most important research and diagnostic tools in medicine, chemistry and physics, taking images of solid objects, determining the structure of crystalline materials, and the chemical composition of even small or hard to reach samples.

The most common source of X-rays for medical applications are X-ray tubes, consisting of a vacuum tube in which electrons, accelerated to energy of about 50 to $60 \mathrm{keV}$, strike a metal target and generate X-rays by bremsstrahlung or X-ray fluorescence.

In the research area, today's most used source of high brightness X-ray beams for studies in biology, chemistry and physics, is the spontaneous radiation from electrons moving in an undulator magnet located in an electron storage ring. A large number of these facilities have been built in many countries, and thousands of scientists use them. The US Advanced Photon Source in the Argonne National Laboratory, the

\footnotetext{
a e-mail: pellegrini@physics.ucla.edu
} 
European Synchrotron Radiation Facility at Grenoble and Spring 8 near Osaka in Japan, are the largest and most powerful. Useful as they are, these facilities have limitations: the shortest X-ray pulse duration is $10-100$ ps; the number of coherent photons, less than one per coherent volume, is small.

$\mathrm{X}$-ray tubes and synchrotron radiation sources are incoherent sources, similar to thermal sources of visible light. In the case of visible light an enormous progress for research and applications has been achieved by the introduction of the laser, generating coherent radiation. How to do the same for X-rays has long been a dream of many scientists. This goal has now been reached with the successful development of the X-ray FEL, which improves the properties of synchrotron radiation sources by orders of magnitude. The radiation is transversely coherent, diffraction limited, and the number of photons in a coherent volume is about $10^{9}$ or larger. The pulse duration changes from 100 to a few femtosecond (fs), with about $10^{13}$ coherent photons/pulse at $100 \mathrm{fs}, 15 \AA$ and $10^{12}$ at $100 \mathrm{fs}, 1.5 \AA$. The number of photons is of course smaller, about $10^{11}-10^{10}$, for pulses only a few femtosecond long. The line width is typically between $10^{-3}$ and $10^{-4}$. These properties are often summarized in a quantity called the photon beam spectral brightness, or sometime simply the brightness, the number of photons per unit area, per unit angular divergence, per second within a line width of $10^{-3}$. The peak spectral brightness measured at the hard X-ray FEL, LCLS, at SLAC [Emma 2009; 2010] and at the soft X-ray FEL, FLASH, at DESY [Faatz 2009], is about 10 and 8 orders of magnitude larger than that of the most powerful synchrotron radiation source, as shown in Figure 1, showing the predicted LCLS performance [Cornacchia 1998] and the experimental results at $0.15 \mathrm{~nm}$. The storage ring based synchrotron radiation sources are again order of magnitudes above other, more conventional, X-ray sources. These are remarkable advances in our ability to explore and study nature, using the powerful tools and techniques provided by X-rays.

By generating X-ray with the characteristics atomic scale length of $1 \AA$ and a time duration of a few to tens of femtosecond, the characteristic time for a valence electron to complete a revolution around the atomic nucleus, the X-ray FEL starts a new chapter in the exploration of the structure and properties of matter at the atomic and molecular level. Determining the structure of non-periodic, non-crystalline, materials in a few femtosecond, or following the evolution of the electron wave function in a chemical reaction on the attosecond time scale, are some of the new, exciting experiments that the X-ray FEL is making possible.

Experiments recently done at LCLS include coherent diffraction imaging of protein nano-crystals [Chapman 2011] and viruses [Seibert 2011], atomic physics [Young 2010], matter under extreme temperature and pressure [Vinko 2011]. A description of the research possible at LCLS, using instruments already available to scientists, is found at http://lcls.slac.stanford.edu/Instruments.aspx. Schneider [Schneider 2010] has recently written an extensive review of experiments at FLASH and other X-ray FELs.

Very recently, in June 2011, a second X-ray FEL, SACLA, built at the Riken Laboratory in Japan, successfully operated at $1.2 \AA$ [Tanaka 2011]. Since then the wavelength range has been extended from $0.63 \AA$ to $2.82 \AA$, with an energy per pulse of about $1 \mathrm{~mJ} 2.8 \AA$ and pulse duration of about $10 \mathrm{fs}$.

In this paper, we describe the history of the successful development of X-ray FELs, leading to the new scientific results just mentioned and the other that will follow in the coming years.

\section{Early history}

Electromagnetic waves are everywhere in the universe. Our life depends on the energy we receive from the Sun in the form of electromagnetic waves. Telecommunications, 

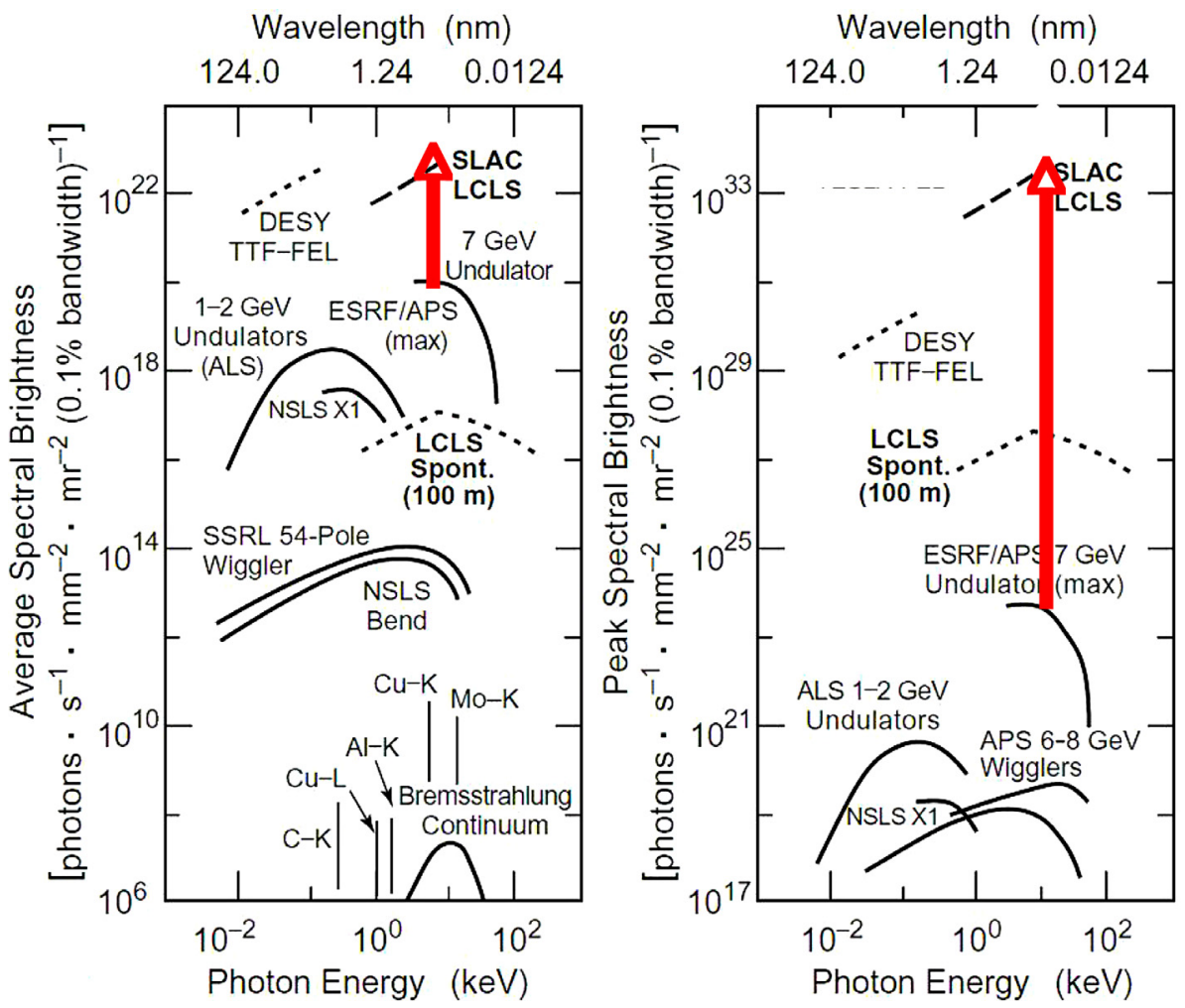

Fig. 1. The average and peak brightness, of photon beams at LCLS, the DESY VUV and soft X-ray FEL, FLASH, and some advanced synchrotron radiation sources. The first results obtained at LCLS are in good agreement with prediction presented in this figure. The red arrows are used to visualize the large increase in brightness obtained at LCLS. Original plots from the LCLS Design Report, reference [3].

in all its forms, are based on the generation of electromagnetic waves. In most cases the electromagnetic waves we generate are incoherent, like those we receive from the Sun, a random superposition of waves at different frequencies and different phases, very much like the noise we hear in a room full of people talking between themselves. However, if instead of people talking at random, we have in the same room a choir the result is dramatically different. The choir members sing at well-defined frequencies or group of frequencies, and emitting sound in phase with each other. The acoustic waves they generate are coherent, having well defined frequencies and being in phase. Doing the same with electromagnetic waves has long been a goal of scientists and engineers, successfully achieved in the early part of the XIX century for radio wavelengths, a few to hundred meters, using the oscillations of electrons in a metal. Unfortunately, this method does not work as well when the wavelength is reduced. New methods and devices have to be invented. Electron beams propagating in vacuum inside a metallic structure have been used, starting in the 1930s, to generate coherent microwaves, at centimeter wavelengths. An article by Pierce [Pierce 1962], one of the scientists contributing to these developments, narrates the history of these developments.

It is interesting at this point to notice, as done by Pierce in his paper, that an important part of the microwave tube history was the invention at Stanford University of the high power klystron by the brothers Sigurd and Varian [Varian 1939]. High power klystrons are the device powering radars, communications systems and, 
of particular interest to our story, particle accelerators, from those used in medical therapy to those used for research in elementary particle and nuclear physics.

William Hansen, Edward Ginzton, Marvin Chodorow, and others, further developed high power klystrons at Stanford University. Their work made possible, and was also stimulated by, the construction on the Stanford campus of a linear electron accelerator, or linac for short, and ultimately by the creation, by Wolfgang Panofsky and his collaborators, of the Stanford Linear Accelerator Center, SLAC, with its two miles long $23 \mathrm{GeV}$ accelerator. As we will see later the two-mile SLAC linac has been a key element that has made possible the development of the X-ray FEL. An excellent review of the early klystron and linear accelerator development is given in the Blue Book, the Stanford Two-Mile Accelerator, a detailed description of the history, operation and technical components of the SLAC linac [Neal 1967]. Douglas W. Dupen wrote the history chapter in the Blue Book. It starts with the following sentence: "The development of linear accelerators at Stanford originated with the late W.W. Hansen interest in X-ray problems in the mid 1930s". For most of its lifetime the SLAC linac has been used to explore, with great success, recognized by several Nobel prizes, the sub-atomic world of leptons and quarks. Its use today to produce X-rays brings its history to complete the circle.

At shorter wavelengths, in the infrared, visible and near UV, wavelengths of about a few micrometers or smaller, generation of coherent radiation has been made possible by the invention of the laser, based on population inversion in an atomic or molecular media, opening a huge field of applications and scientific exploration. There are many papers on the history of the lasers and their development, among them those by Mario Bertolotti [Bertolotti 2005] and by Zinth et al. [Zinth 2011]. Scientists have since utilized the laser as a powerful instrument for research in all areas of science. Laser operating in the near infrared can generate pulses with extremely large peak power, up to terawatts or even petawatts, and extremely short duration, as short as a femtosecond or even less, characteristic of some atomic and molecular systems. One femtosecond (fs) is the time one valence electron takes to go around one nucleus.

However the space resolution is limited by the radiation wavelength to the micrometer or sub-micrometer range, well above the atomic scale length of about $1 \AA$. Extending atomic lasers to the Angstrom region is made very difficult by the huge amount of power needed to produce population inversion in the inner atomic electronics level corresponding to this wavelength. Finding a way to have coherent radiation sources that jointly have atomic space and time resolution, generating X-ray pulses with femtosecond duration and Ångstrom wavelength, an X-ray laser, would open a new way to explore the spatial structure and dynamics of atomic and molecular processes, a real breakthrough for the study of the properties of matter.

The dream of an X-ray laser has motivated many scientists for many decades. This paper describes how, using free-electron lasers, based on relativistic electron beams propagating in vacuum in a particular type of magnet, an undulator, this dream has become reality.

\section{From microwave tubes to FELs}

Microwave tubes, and in particular klystrons, developed initially during World War II to power radars, were the first sources of coherent electromagnetic radiation from free-electron beams. The term free-electron describes the fact that, contrary to the case of atomic lasers, the electrons are not bound to a nucleus in an energy level. They make use of a metallic slow wave structure to produce an electric field parallel to the direction of propagation of an electron beam and make the phase velocity 


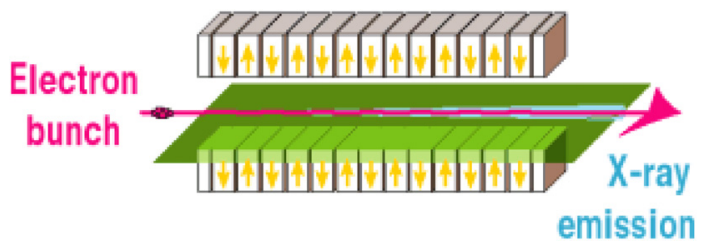

Fig. 2. An electron bunch moves through a planar undulator magnet. Its trajectory oscillates around the longitudinal magnet axis, in a plane perpendicular to the magnetic field. The electron acceleration, perpendicular to the axis, is periodic with the undulator magnet period.

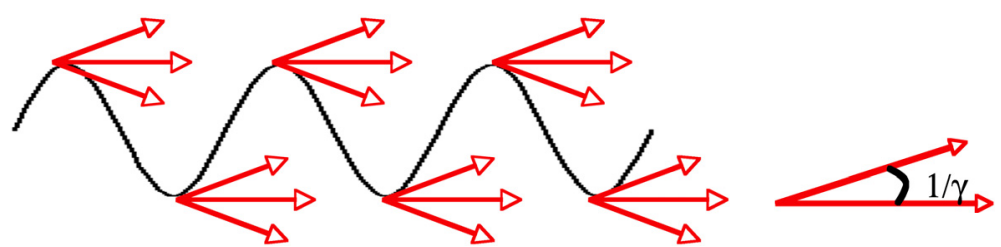

Fig. 3. Electron trajectory and emitted radiation. The radiation is emitted in the forward direction, within an angle $1 / \gamma$, where $\gamma$ is the electron energy in rest mass units, $E=m c^{2} \gamma$, and mostly near the part of the trajectory where the acceleration is larger.

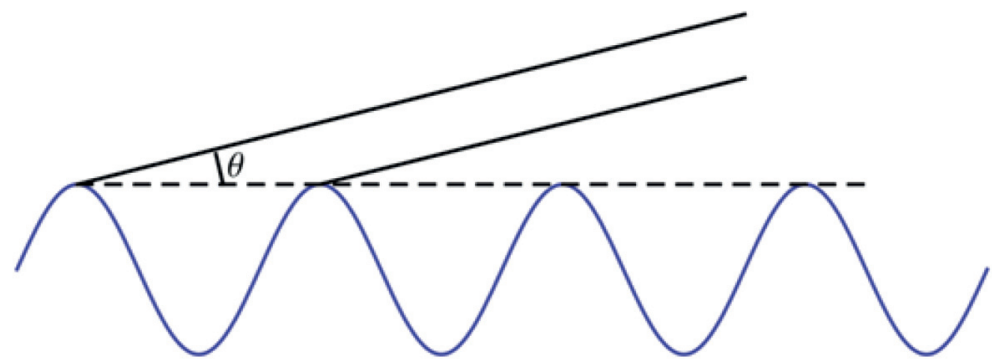

Fig. 4. The interference between the waves emitted from each undulator period is positive when the condition (1) is satisfied.

of the electric field smaller than that of light. A resonant energy exchange between electrons and the radiation is obtained when the electron velocity and the wave phase velocity are nearly equal. This condition requires the size of the structure to be of the order of the radiation wavelength, limiting the tubes, typically, to centimeters long wavelength.

A different approach to reach shorter wavelengths was proposed in 1951 by Motz [Motz 1951], using a relativistic electron beam propagating through an undulator magnet to produce electromagnetic radiation. In the simplest case, shown in Figure 2, an undulator consists of a magnet array, with alternating North-South polarities, generating a field, transverse to system axis, of maximum value $B_{0}$ and oscillating in a plane like a sinusoid. In this field an electron with a component of the velocity parallel to the undulator axis follows a sinusoidal trajectory around the same axis, in a plane perpendicular to the direction of the magnetic field, as shown in Figure 3.

Being accelerated, the electron emits a wave train with a number of waves equal to the number of magnetic periods, $N_{U}$. The far field radiation seen by an observer on the undulator axis, $z$, at some distance from the undulator, is the result of interference between the waves emitted at each period, as shown in Figure 4. The interference gives 
1 a positive addition of the waves when the time delay between the waves emitted at

2 two consecutive electron oscillation maxima is equal to the wavelength

$$
c \Delta T=\frac{\lambda_{U}}{\beta_{z}}-\lambda_{U} \cos \theta=\lambda
$$

where $\beta_{z}$ is the longitudinal velocity, velocity along the undulator axis. in units of the light velocity, $c$. We assume the longitudinal velocity to be near to one, $\beta_{z} \approx 1$. From this equation we obtain the relationship

$$
\lambda=\lambda_{U}\left(\frac{1}{\beta_{z}}-\frac{1}{\cos \theta}\right)
$$

between the radiation wavelength $\lambda$, the undulator period, $\lambda_{U}$, and the electron longitudinal velocity. For relativistic electrons it is possible to assume

$$
\theta \leq 1 / \gamma \ll 1, \quad 1-\beta_{z} \ll 1 .
$$

In this case the radiation wavelength is much shorter than the undulator period.

The electron trajectory is determined by the undulator magnetic field, and has a component, $\vec{V}_{T}$, transverse to the undulator axis. The motion in the undulator magnetic field leaves the electron energy, and the magnitude of its velocity, constant, when we neglect the emission of radiation, a quite good first approximation. The transverse component of the velocity oscillate with the period, $\lambda_{U}$, of the undulator field

$$
V_{T}=(K / \gamma) \sin \left(2 \pi z / \lambda_{U}\right)
$$

where $z$ is the position along the undulator axis and

$$
K=e B_{0} \lambda_{U} / 2 \pi m c^{2}
$$

is a quantity characterizing the undulator magnet, called the undulator parameter, typically of the order of one. For relativistic electrons $\gamma \gg 1$, the transverse velocity is small, $K / \gamma \ll 1$, and the axial electron velocity is constant to second order in this quantity. The velocity and the energy $\gamma$ are related by $\beta_{x}^{2}+\beta_{z}^{2}=1-1 / \gamma^{2}$. Using this relationship and the smallness of the transverse velocity, the relationship (2) between the undulator period and the radiation wavelength can be rewritten in the most widely used form

$$
\lambda=\lambda_{U}\left(1+K^{2} / 2+\gamma^{2} \theta^{2}\right) / 2 \gamma^{2} .
$$

This dependence makes it easy to change the wavelength from centimeters or millimeters to Ångstroms by varying the electrons energy from a few $\mathrm{MeV}$, to about $10-20 \mathrm{GeV}$.

There are several books and reviews on the physics and technology of FELs, where the characteristics of the undulator magnetic field, the electron trajectories and the emitted radiation are discussed in detail. The interested reader can see for instance the review paper by [Pellegrini 2004], where other references are given.

The line width of the emitted radiation is approximately the inverse of the number of waves in the emitted wave train

$$
\frac{\Delta \lambda}{\lambda} \approx \frac{1}{N_{U}}
$$

As an example let us consider a case similar to that of LCLS. Assuming $\gamma=3 \times 10^{4}$, $\lambda_{U}=3 \mathrm{~cm}, K=3$, we obtain $\lambda \sim 1 \AA$. 

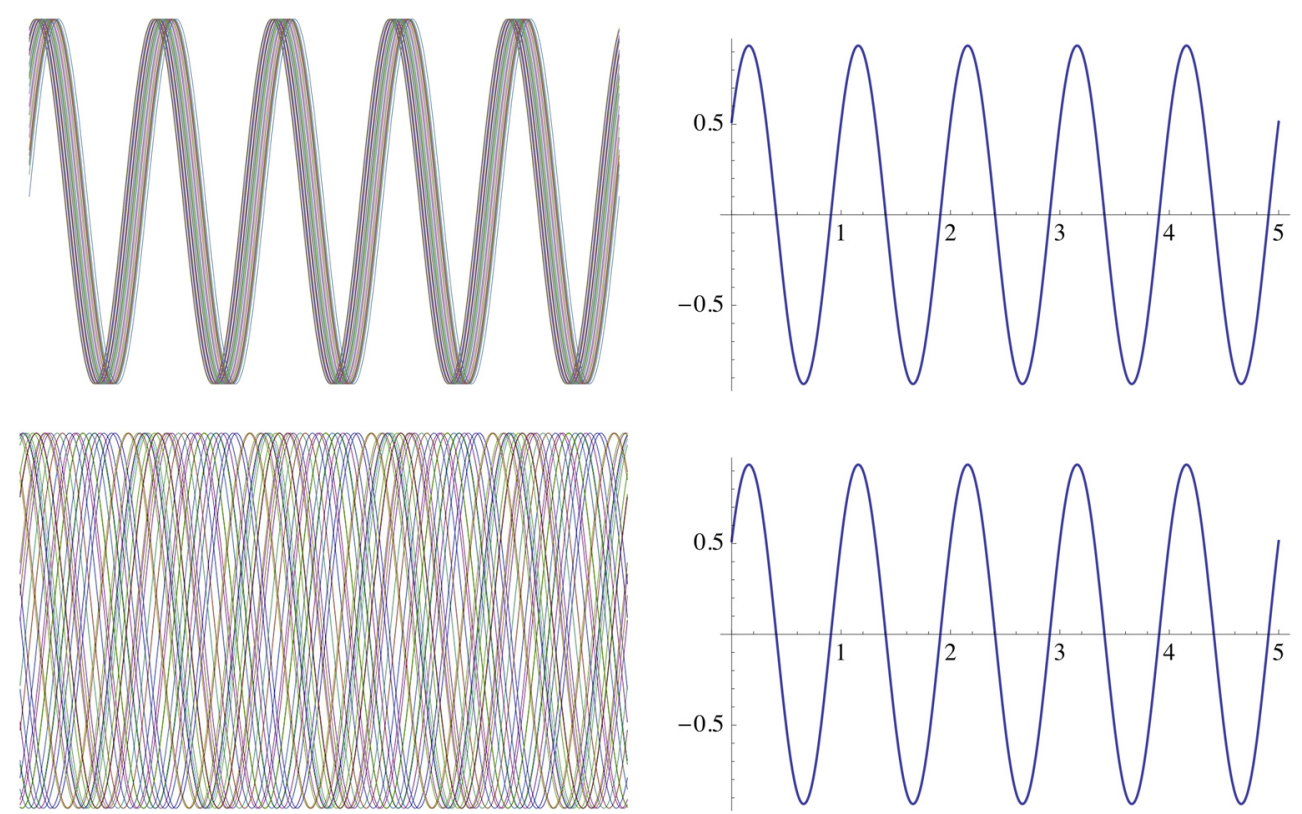

Fig. 5. The superposition of the electromagnetic waves emitted by 30 relativistic electrons crossing an undulator magnet for case a, top, and b, bottom. Top left, waves superposition when the phase is a random number between 0 and $2 \pi$, top right average field amplitude. For a single wave the amplitude is normalized to 1 . Bottom left and right, wave superposition and average amplitude when the phase is a random number between 0 and $\pi / 5$. Notice the larger value of the average amplitude in case a.

The radiation generated by a beam with $N_{e}$ electrons, distributed over a certain length $L_{B}$, crossing an undulator, is the superposition of the wave trains generated by each electron. The result of the superposition depends on the radiation wavelength, the electron bunch length, and the details of the electron longitudinal distribution, because the wave trains generated by electrons entering the undulator at different times have a phase difference proportional to the difference of their arrival time at the undulator entrance. The total radiation intensity depends on how the wave trains superimpose.

The simplest case, (a) is when the bunch length is much shorter than the radiation wavelength. In this case all the wave trains have approximately the same phase and the radiation intensity is proportional to the square of the electron number, $I \sim N_{e}^{2}$, the radiation from all the electrons is coherent, the radiation wave front is like that generated by a single super-particle of charge $N_{e}$.

If the electron bunch length is much larger than the radiation wavelength, and the longitudinal electron distribution is random over the scale defined by the radiation wavelength, case (b), the superposition is similar to that shown in Figure 5, top case. In this case the intensity is proportional to the number of electrons. The difference in intensity between the two cases is rather large, since typically the number of electrons in a bunch can be as large as $10^{9}$ to $10^{10}$.

On the other hand, while it is within the range of our technologies to easily generate in electron accelerator bunches with this number of particles and lengths of centimeters to millimeters, it becomes increasingly difficult to do so at sub-millimeter to micro-meter lengths. Hence the interest in another case, case (c), with bunch length much longer than the wavelength, but electrons organized in micro-bunches separated by about one radiation wavelength. The wave trains are nearly in phase and 
the radiation intensity can be again proportional to some power of $N_{e}$ intermediate between 1 and 2, depending on how well the condition is satisfied.

In case (a) the radiation is longitudinally coherent, while in case (b) it has the characteristics of a thermal source. Case (c) is intermediate between the other two.

This discussion can be made quantitative characterizing how the wave trains superimpose with a quantity called the bunching factor

$$
B=\frac{1}{N_{e}} \sum_{n=1}^{N_{e}} \exp \left(2 \pi i z_{0 n} / \lambda\right)
$$

where $z_{0 n}$ is the initial position of the electrons within the bunch. The bunching factor is the Fourier component of electron longitudinal distribution at the radiation frequency, which is also proportional to the Fourier component of the longitudinal charge density. The transverse electric current, the source term for the electromagnetic field, is also proportional to the bunching factor. The radiation intensity is proportional to the square of the bunching factor and is zero if the bunching factor is zero, as is the case for a beam with a uniform electron distribution. Evaluating $|B|^{2}$, which changes between zero, in the case of a uniform particle distribution, and one, for particles at the same position or separated by the wavelength $\lambda$, leads to the results discussed before.

Since case (b) is the most common when we consider short wavelengths, nanometer or fraction of a nanometer, finding a way to make a transition from (b) to (c) can be most important and give a very large increase in radiation intensity. As we will see the FEL gives us a way to do exactly that.

\section{Early experiments and the small signal gain theory}

Motz followed his 1951 paper with experimental work. About two years later, in 1953, he reported [Motz 1953] the results of an experiment using an electron beam of a linear accelerator at Stanford University. He observed incoherent visible light for electron beam energy of about $100 \mathrm{MeV}$, and coherent emission, at a radiation wavelength of a few $\mathrm{mm}$, with an electron beam energy of 3 to $5 \mathrm{MeV}$. The typical bunch length for the linac beam used by Motz is about one $\mathrm{mm}$, so in the high-energy case it is much longer than the radiation wavelength, and the emitted wave trains superimpose as in case (b). For the low energy case we are approximately in case (a).

Philips [Philips 1960] in 1960 developed another device, the Ubitron, using the interaction between an electron beam executing periodic transverse oscillations in a magnet and the $\mathrm{TE}_{01}$ mode in an unloaded waveguide.

Madey introduced the free-electron laser (FEL) concept in 1971 [Madey 1971]. It consists, as shown in Figure 6, of a linear accelerator, an undulator magnet, and an input electromagnetic wave, which generates stimulated emission from the electrons, amplifying the input wave. As we will see later the amplification process can also be described as a transition of a beam initially in case (b), with a random distribution of electrons on the wavelength scale, to a beam corresponding to case (c), with a large increase in the bunching factor and thus in the intensity of the radiation field.

Madey experimental system is shown in Figure 6 [Elias 1976]. The undulator is helical, consisting of bifilar helical coils with current flowing in each coil in opposite directions. It generates a rotating transverse periodic magnetic field that, near the axis, is

$$
\begin{aligned}
& B_{x}=B_{0} \cos \left(2 \pi z / \lambda_{U}\right) \\
& B_{y}=B_{0} \sin \left(2 \pi z / \lambda_{U}\right) .
\end{aligned}
$$




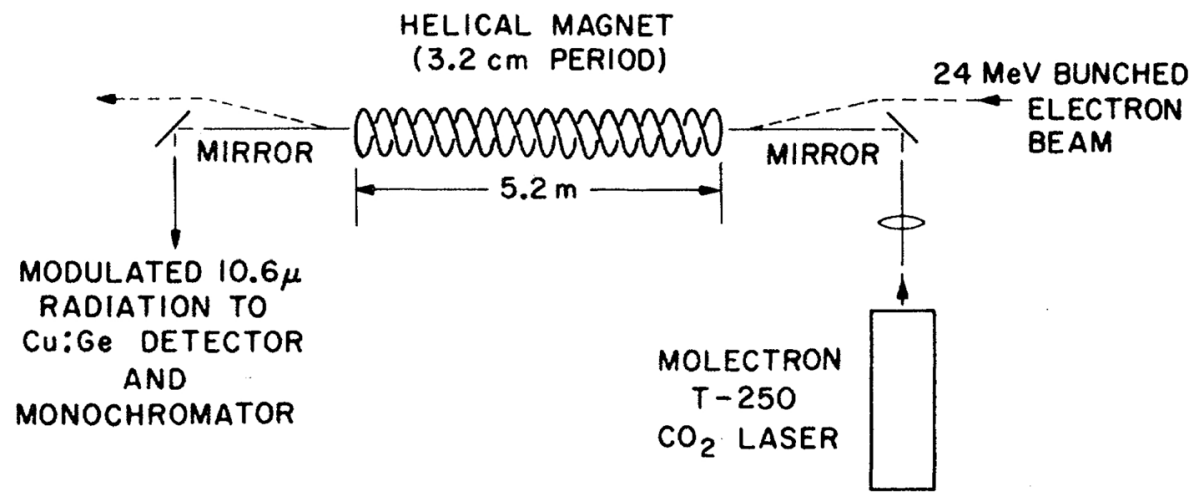

Fig. 6. Madey's amplifier experiment. The undulator is a bifilar superconducting coil (from ref. [Elias 1976]).

In this field the trajectory of an electron of energy and longitudinal velocity $\gamma, \beta_{z}$ is a helix of radius $a=\lambda_{U} K / 2 \pi \gamma \beta_{z}$. The electron velocity transverse to the undulator axis is

$$
\begin{aligned}
& \beta_{x}=(K / \gamma) \cos \left(2 \pi z / \lambda_{U}\right) \\
& \beta_{y}=(K / \gamma) \sin \left(2 \pi z / \lambda_{U}\right)
\end{aligned}
$$

while the longitudinal velocity $\beta_{z}$ is a constant $\beta_{z}^{2}=1-1 / \gamma^{2}-K^{2} / \gamma^{2}$.

For a relativistic electron the longitudinal velocity can be well approximated by

$$
\beta_{z}=1-\left(1+K^{2}\right) / 2 \gamma^{2} .
$$

While there are differences between a helical and a planar undulator, for instance the radiation is circularly polarized instead of linearly polarized, the basic physics of emission of radiation by the electrons is the same, and the formula (4) for the radiation wavelength is still valid with the simple substitution of $K^{2} / 2$ with $K^{2}$ [Murphy 1990]. The interaction of the electrons with the undulator and radiation field, the FEL mechanism, is also essentially the same, with some minor differences, in the two cases. In the following description of the FEL theory we will consider the somewhat simpler case of a helical undulator.

Madey described the stimulated radiation emission process using a quantum mechanical approach, in the reference frame where the electrons have zero longitudinal velocity, and execute an oscillation in the transverse direction. Using the WeiszäckerWilliams approximation [Weiszäcker 1934; Williams 1935] the undulator field is represented as a circularly polarized plane wave. In this frame the system consists of two electromagnetic waves traveling in opposite directions, and electrons oscillating transversely to the direction of propagation of the waves. The small signal gain is evaluated from the photon transition probability from one state to the other, and the corresponding change in intensity of the input wave. The condition to obtain gain is that the frequency of the input signal be near to that of the spontaneous undulator radiation.

Madey also considers over what wavelength range the FEL would work, and he reaches the conclusion: "The dependence of the gain on the square of the final state wavelength probably precludes the development of steady state oscillations in the region beyond the ultraviolet. However, it remains possible that, considering the intensity, the spontaneously emitted radiation might itself find applications". 
A very interesting aspect the theory is that the gain, even if evaluated using the quantum theory of radiation, does not depend on Planck's constant, the effect is classical. The need of a quantum theory and the limitations of a classical theory were studied in the early 1980s [Bosco 1983; Becker 1982; 1983; Dattoli 1985], reaching the conclusion that the effect is classical to very good approximation if the electron recoil in the emission of a photon is small. More precisely the ratio of the photon energy to the electron energy must be less than the radiation line-width, given by (5). This is certainly the case for infrared, visible, UV FELs, and even for most X-ray FELs.

Colson [Colson 1977] developed the classical theory of the FEL in the small gain approximation. The basic physics, in the classical description, is the energy exchange between the electron beam and the electromagnetic plane wave. The electron trajectory is determined, to a very good approximation, by the undulator magnetic field, and has a component, $\vec{V}_{T}$, transverse to the undulator axis, which is also the direction of propagation of the wave. The motion in the undulator magnetic field leaves the electron energy, and the magnitude of its velocity, constant. The transverse components oscillate with the period, $\lambda_{U}$, of the undulator field, as in equation (3) or (8). For relativistic electrons, $\gamma \gg 1$, the traverse velocity is small, $K / \gamma \ll 1$, and the axial electron velocity is constant to second order in this quantity.

The electric field of the input wave is also transverse to the undulator axis, and is chosen to be parallel to the transverse component of the velocity. Its amplitude is given by

$$
\begin{aligned}
& E_{R, x}=E_{R 0} \sin \left[2 \pi(z-c t) / \lambda_{R}\right] \\
& E_{R, y}=E_{R 0} \cos \left[2 \pi(z-c t) / \lambda_{R}\right] .
\end{aligned}
$$

The electron energy change is then given by

$$
m c^{2} d \gamma / d t=e \vec{V}_{T} \cdot \vec{E}_{R}=\left(e E_{R 0} K / 2 \gamma\right) \sin \Phi
$$

where

$$
\Phi=\frac{2 \pi z}{\lambda_{U}}+\frac{2 \pi(z-c t)}{\lambda_{R}}
$$

the FEL phase, is the relative phase of the electron oscillation in the undulator and the input wave.

Assuming $z=\beta_{z}$ ct we can evaluate the time derivative of the FEL phase

$$
\frac{d \Phi}{d t}=\frac{2 \pi c \beta_{z}}{\lambda_{R}}\left\{\frac{\lambda_{R}}{\lambda_{U}}-\frac{1-\beta_{z}}{\beta_{z}}\right\} .
$$

The energy exchange averages out to zero except when the derivative of the phase is zero or small. The phase is then constant or slowly changing while the electron moves through the undulator, as shown in Figure 7.

The condition for constant phase requires that, when the electron moves ahead by one undulator period its oscillation phase changes by $2 \pi$, the electric field phase also changes by the same amount, or $\left(\lambda_{U} / \lambda_{R}\right)\left(1-\beta_{z}\right) / \beta_{z}=1$. This is the same condition as obtained in (2) for spontaneous emission peak in the forward direction, $\theta=0$, and can also be written as in (4)

$$
\lambda_{R}=\lambda_{U}\left(1+K^{2}\right) / 2 \gamma^{2} .
$$

We can use the condition of constant phase to define, for a given radiation wavelength, the resonant energy, as the electron energy for which (13) is satisfied, or

$$
\gamma_{R}^{2}=\lambda_{U}\left(1+K^{2}\right) / 2 \lambda_{R} .
$$




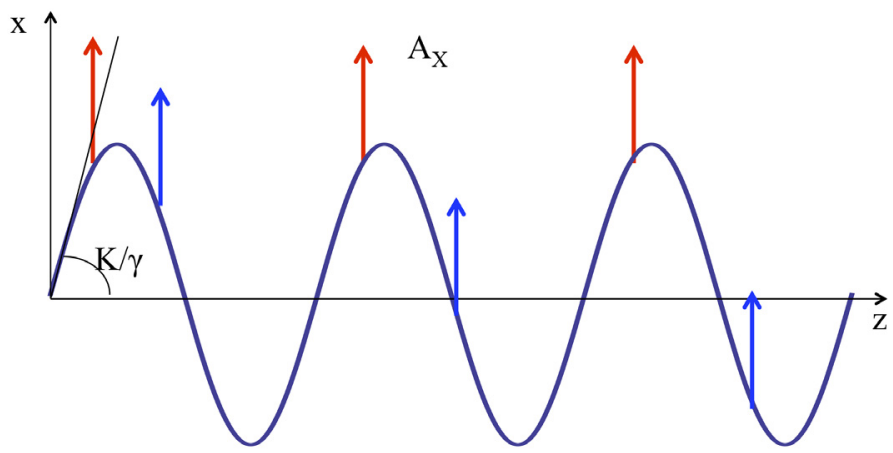

Fig. 7. The plane wave electric field component along the $x$-axis - red and blue vectors - is parallel to the $x$ component of the electron velocity. The electron trajectory in the undulator magnetic field, pointing in the direction perpendicular to the $x-z$ plane, is a sinusoid. The $z$-axis is the undulator axis. In the case of the red electric vector the phase between the electron transverse velocity and the electric field remains constant, giving a large electron energy change. In the other case, blue vectors, the phase is changing and the average value of the electron energy change is nearly zero.

Colson's paper studies the electron energy change in the small signal case limit, assuming that the change in the radiation field amplitude is small and can be neglected when solving the coupled system of equations (10) and (12). The change in the radiation field energy is then assumed to be equal and opposite to that of the electron beam. The last step is the evaluation of the gain $G=\Delta I / I$, from the change of the radiation field energy $I$.

In the small gain approximation, the electron dynamics in the combined field of the undulator magnet and a plane wave co-propagating with the beam is the same as that of an ensemble of pendulums. In fact linearizing equations (10), (12) and introducing the new variables $\eta=\left(\gamma-\gamma_{R}\right) / \gamma_{R} \ll 1, \tau=4 \pi z / \lambda_{U}$, the change in the electron energy and FEL phase is described by [Colson 1977]

$$
\begin{aligned}
& \frac{d \eta}{d \tau}=\Omega^{2} \sin \Phi, \\
& \frac{d \Phi}{d \tau}=\eta
\end{aligned}
$$

where the small oscillation frequency is

$$
\Omega^{2}=\frac{e A K \lambda_{U}}{4 \pi \gamma_{R}^{2}} \ll 1 .
$$

These equations are the same as that of a pendulum in a constant gravitational field. The small amplitude frequency is proportional to the electric field amplitude. The dynamics of the electron can be simply shown with a plot in the energy-phase plane. The trajectories in this plane are shown in Figure 8.

Energy can be transferred from the electrons to the radiation field, or vice versa, depending on the initial beam energy, or, more precisely, on the quantity

$$
\delta=\frac{\gamma-\gamma_{R}}{\gamma_{R}}
$$

called the detuning parameter. For $\delta=0$ the beam energy is such that the FEL phase does not change, and the spontaneous radiation wavelength is exactly equal to the radiation field wavelength. 


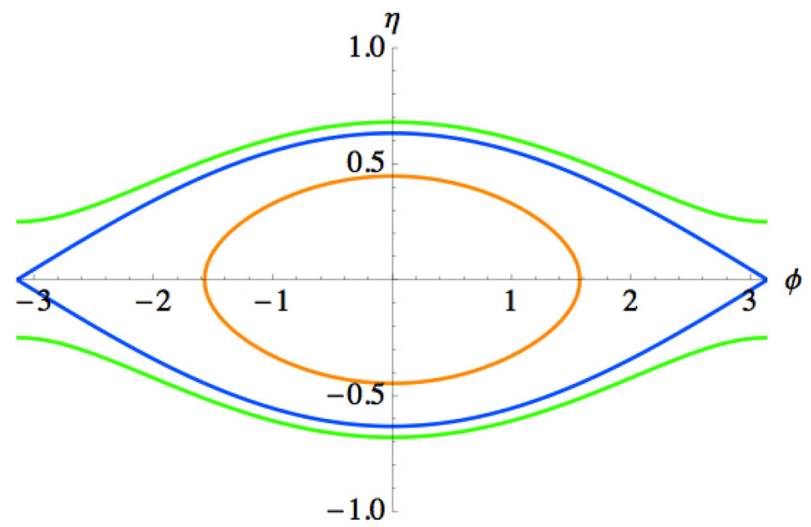

Fig. 8. Phase space plane for electrons in the combined undulator-radiation field potential.
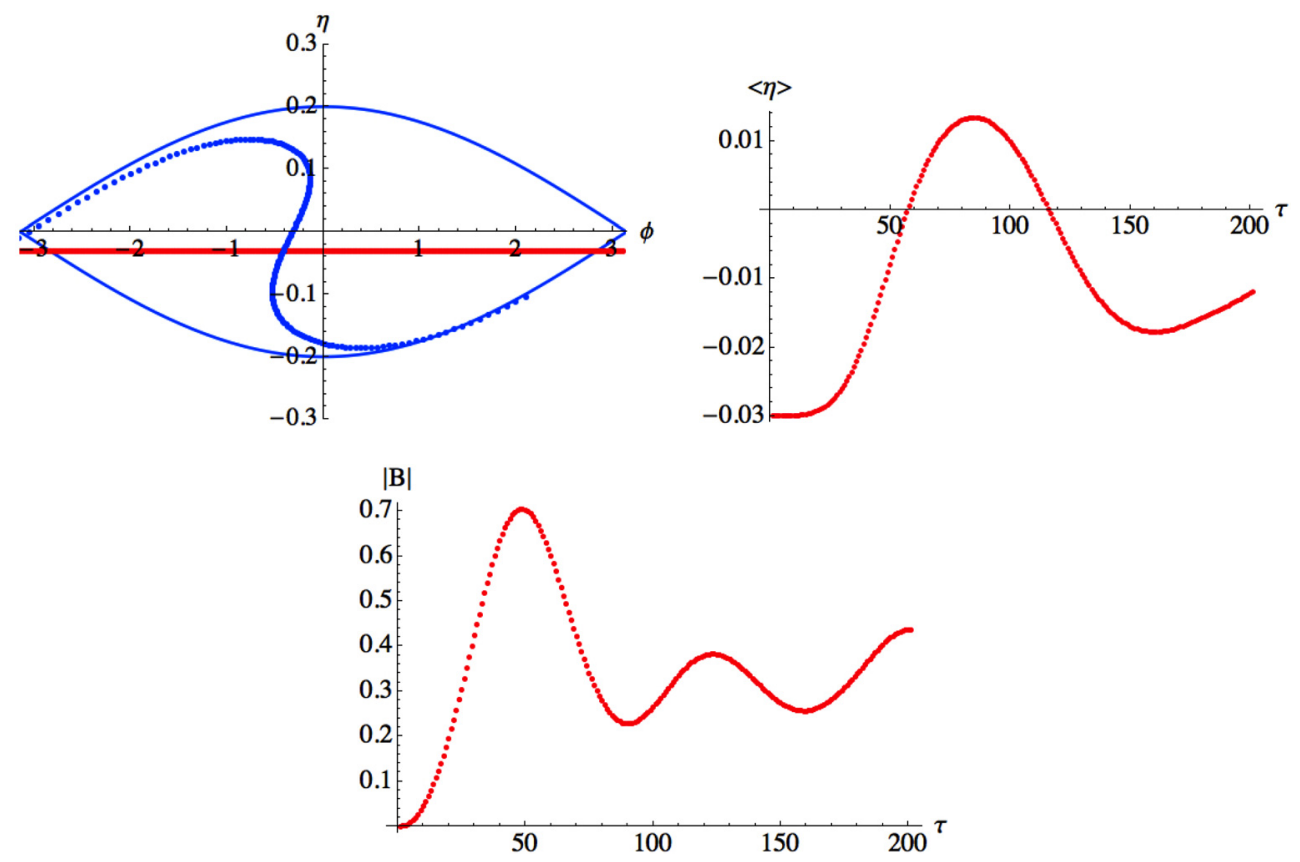

Fig. 9. Evolution of a monochromatic electron beam in the undulator radiation field potential. Left plot: phase space change from the initial distribution, red line, to a point along the undulator where the bunching factor is large. Right plot: average beam energy along the undulator axis, $z$. Bottom plot: bunching factor evolution. The initial electron energy is smaller than the resonant energy. The electrons gain energy along the undulator, corresponding to negative gain, or electron acceleration.

The dependence of the gain on the detuning, and other undulator and electron 2 beam parameters, for a monochromatic electron beam, is given by

$$
G_{0}=4 \sqrt{2} \pi^{2} \lambda_{r}^{3 / 2} \lambda_{U}^{1 / 2} \frac{K^{2}}{\left(!+K^{2}\right)^{3 / 2}} \frac{I_{P}}{I_{A} \Sigma} N_{U}^{3} F\left(4 \pi N_{U}\left(\gamma_{0}-\gamma_{R}\right) / \gamma_{R}\right)
$$

3 where $I_{P}$ is the peak electron beam current, $\Sigma$ its transverse area and $I_{A}=e c / r_{e} \approx$ $417000 \mathrm{~A}$ is the Alfven current ( $r_{e}$ is the classical electron radius). 

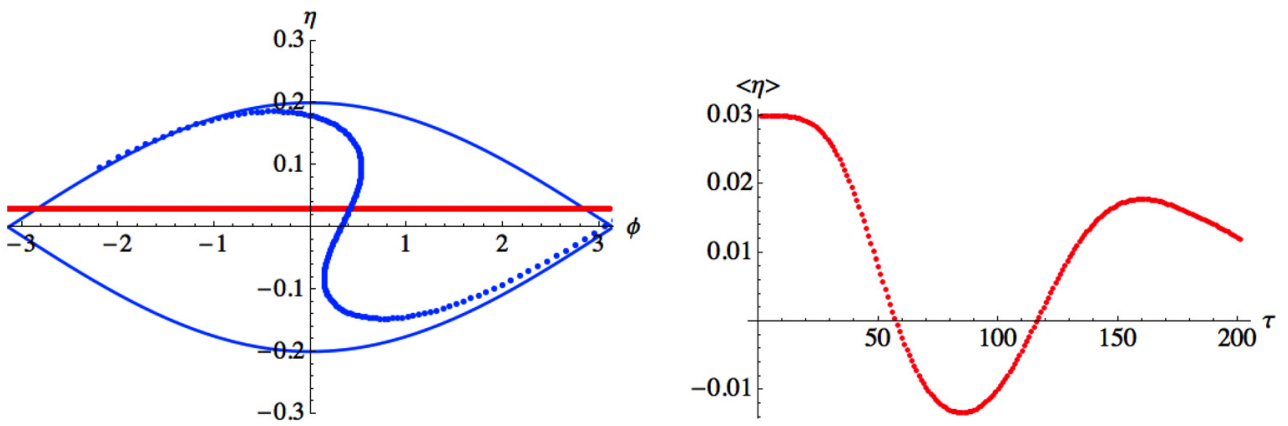

Fig. 10. The initial electron beam energy is larger than the resonant energy. Electrons lose energy along the undulator and the radiation field intensity increases, corresponding to positive gain, or electron deceleration. The evolution of the bunching factor is the same as in the previous figure.

For a beam with an energy spread the gain function must be folded with the electron energy distribution. If the energy distribution covers both sides, positive and negative of the gain curve the result is zero or nearly zero. To have good gain the energy spread must be smaller than the width of the gain curve, giving the condition

$$
\frac{\Delta E}{E}<\frac{1}{N_{U}}
$$

This is the first example of the requirements that the electron beam must satisfy for the FEL to have gain and work as a laser. By inspecting the gain formula (18) one can also see that for the gain to be large enough to be of practical value the ratio $I_{P} / \Sigma$, which means the electron density, must also be large and remains so when the electrons propagate through the undulator. This leads to a requirement of focusing along the undulator and on the electron angular spread. We will discuss in more detail the importance of these conditions in the next section on High Gain FELs, and how they reflect on the accelerator chosen to generate the electron beam.

Madey's group demonstrated the FEL feasibility in two experiments. The first [Elias 1976], shown in Figure 6, used a $24 \mathrm{MeV}$ electron beam from a superconducting linear accelerator at Stanford, with current of 5 to $70 \mathrm{~mA}$. The helical undulator had a period of $3.2 \mathrm{~cm}$ and a length of $5.2 \mathrm{~m}$. The radiation wavelength was $10.6 \mu \mathrm{m}$ and the single pass gain was as large as 7\%. The second experiment [Deacon 1977], shown in Figure 12, was an oscillator, at a wavelength of $3.4 \mu \mathrm{m}$, using the same helical undulator surrounded by an optical cavity $12.7 \mathrm{~m}$ long and an electron beam energy of $43 \mathrm{MeV}$. The cavity length is chosen so that the back and forth travel time of the light pulse in the cavity is equal to the time separation between electron bunches from the linac. The beam peak current was $2.6 \mathrm{~A}$ and the mirror cavity transmission $1.5 \%$. When the current is increased near the maximum value, one observed a narrow radiation line, with peak power of about $7 \mathrm{~kW}$.

When the input signal is an external field the FEL is in an amplifier configuration. The initial signal can also be the undulator radiation generated by the beam itself, in which case the system is called a self amplified spontaneous emission (SASE) amplifier. The oscillator can also start from the spontaneous radiation, or an external input.

As precursors to his own work Madey quotes the work of Motz already mentioned, and that of Pantell et al. [Pantell 1968] on stimulated Compton scattering. Other physicists exploring similar ideas were Robert Palmer [Palmer 1972], Robinson [Robinson 1985], whose paper was published posthumous, and Csonka [Csonka 1978a; 1978b]. 


\section{The high gain theory and SASE}

Madey's experiments had a great impact, attracting the attention of many people. By that time, the laser, based on population inversion and stimulated emission from atoms and molecules, had been invented and developed, starting from the late 1950s and the1960s [Bertolotti 2005; Zinth 2011]. Lasers operate mainly in the visible and infrared spectral region generating high peak (TW), and average $(\mathrm{kW})$ power. They can generate femtosecond and even attosecond pulses. Extending their wavelength to the atomic scale, Angstrom, region is however, difficult, because of the very high pump power, scaling like the inverse of the fourth power of the photon frequency, needed to produce population inversion.

The FELs, notwithstanding their larger size, cost and complexity, offer some advantages over the atomic/molecular lasers: operation over a large wavelength range, ease of changing the wavelength, capability of very high average power, and a more favorable scaling for the gain at short wavelengths. Initially the main research emphasis for FELs was the development of high average power, MW level, infrared systems for defense applications. Strong financial support was given in the US to this research as part of the Strategic Defense Initiative. A brief history of the FEL development for the Strategic Defense Initiative can be found in the report of a committee of the National Academy Press chaired By Katsouleas [Katsouleas 2009].

In the late 1970s and early 1980s the FEL theory was developed beyond Madey's small signal gain case. In the small signal case theory the electric field is kept cons tant during the interaction, and at the undulator exit the electron energy loss is given to the radiation field, the theory is not self consistent. The next step was to formulate a fully self-consistent theory, including the evolution of the electromagnetic field during the interaction, in a single undulator pass of the electron beam, so that the electromagnetic field changes together with the electron beam distribution [Kroll 1978; Sprangle 1980; Kondratenko 1980; Gover 1981; Dattoli 1981; Bonifacio 1982; 1984; Gea-Banacloche 1984; Sprangle 1985; Jerby 1985; Kim 1986a; Wang 1986; Bonifacio 1987]. This analysis leads to some very interesting results. The most important is that beyond the small signal gain case there is a high gain regime where the electromagnetic field of the amplified radiation grows exponentially during one undulator pass, until it reaches a large saturation value.

The paper by Saldin and Kondratenko [Kondratenko 1980] is an important contribution. In this paper it was considered, for the first time, the possibility of using the high gain regime, starting from spontaneous radiation, to reach saturation in a single pass infrared FEL, using low energy electron beams (a few to $10 \mathrm{MeV}$ ), eliminating the need of an optical cavity. This paper opened the possibility of using the same approach at shorter wavelengths, as we will discuss in the next sections. In a second paper [Derbenev 1982], in 1982, they again discussed the case of infrared FELs and considered the possibility of increasing the beam energy to about $1 \mathrm{GeV}$ to produce soft X-rays.

The one-dimensional high gain theory of a SASE-FEL developed by Bonifacio et al. [Bonifacio 1984] provides a very useful picture of the FEL process, describing all FEL physics, including the start from an external signal or from the spontaneous radiation noise, exponential gain length, saturation power and undulator saturation length, with one single quantity, the FEL parameter, $\rho$. This parameter is a function of the electron beam density and energy, and of the undulator period and magnetic field. Besides clarifying some of the fundamental physics of the system, it gives a simple way to evaluate and analyze the FEL characteristics on the back of an envelope.

The exponential growth is due to a collective effect, a phenomenon of selforganization of the electron beam that leads from an initial state with a random longitudinal distribution of the electron beam to a state in which the electrons are 
organized in micro-bunches separated by the radiation wavelength. In other words it takes the electron beam from the case (b) considered before to case (c). If we assume a long enough undulator and propagate an electron beam with the proper characteristics through it, the electron beam longitudinal distribution evolves from one which is random on the scale length of the radiation wavelength to one in which the electron are distributed on parallel planes separated by one wavelength, a kind of 1-dimensional crystal.

The process occurs in three steps:

1. The interaction of the electrons with the electromagnetic wave in the undulator creates an electron energy modulation on the scale of the radiation wavelength $\lambda$; notice that the electromagnetic wave can be an external laser field, as in the amplifier experiment discussed before, or a wave generated by the spontaneous emission process.

2. The energy modulation leads to electron bunching, because electron with a larger energy are less bent in the undulator magnetic field, and the length of their trajectory is shorter than that of an electron with a smaller energy, as shown in Figure 13.

3. A larger bunching factor B leads to higher EM field intensity; going back now to step 1 we see that we have a system with positive feedback, generating a collective instability.

1

2

3

4

\section{5} 6

The process can be completely characterized by the FEL parameter [Bonifacio 1984],

$$
\rho=\left(\frac{K}{4} \frac{\Omega_{p}}{\omega_{U}}\right)^{2 / 3}
$$

where $\Omega_{p}=\left(4 \pi n_{e} r_{e} c^{2} / \gamma^{3}\right)$ is the beam plasma frequency, $n_{e}$ is the electron bunch density and $\omega_{U}=2 \pi c / \lambda_{U}$. The FEL parameter gives:

1. The gain length

$$
L_{G}=\lambda_{U} / 4 \sqrt{\pi} \rho
$$

characterizing the exponential growth of the radiation power $P_{L}=P_{0} \exp \left(z / L_{G}\right) \quad 26$ along the undulator axis, $z$.

2. The saturation power of the radiation field

$$
P_{S}=\rho E I_{P}
$$

$E$ being the electron beam energy and $I_{P}$ its peak current. Hence the FEL parameter gives the fraction of the beam power transferred to the radiation field. Since the radiation pulse duration and the bunch duration are approximately equal, $\rho$ also measures the fraction of the beam energy given to the radiation field.

3. When the amplification noise starts from the spontaneous radiation the FEL parameter also gives the undulator length needed to reach saturation

$$
L_{S} \approx 20 L_{G} \approx \lambda_{U} / \rho
$$

a number of undulator periods about equal to the inverse of $\rho$. From (5) one can also see that the FEL parameter gives the radiation pulse line width. Since the number of undulator periods, and thus the undulator length, required to reach saturation is of the order of $\rho$, it is necessary to have a system of practical utility that the FEL parameters be larger than 1/10000.

The analysis of the coupled system of the electromagnetic field and electrons moving in the undulator field done in [Bonifacio 1984] can also be found in more details 


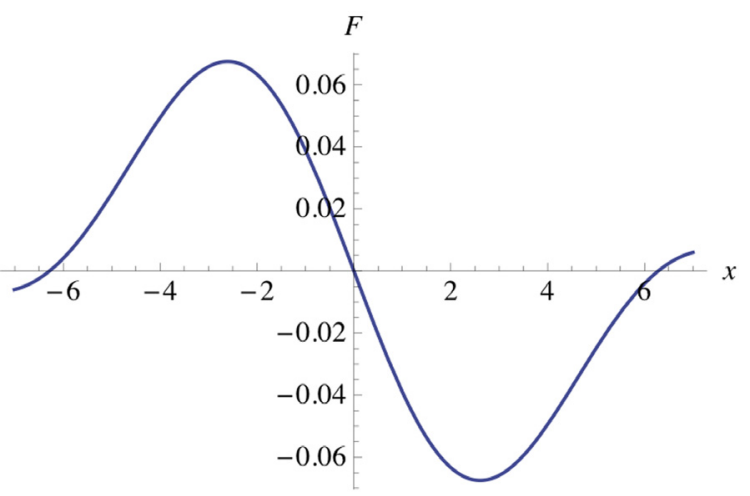

Fig. 11. The small signal gain curve, giving the gain amplitude as a function of, where $\delta$ is the detuning parameter defined in (17). The gain can be positive or negative, energy can be transferred from the electrons to the radiation or vice versa.

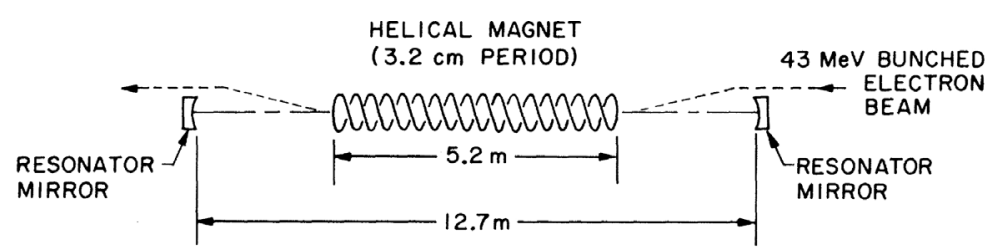

FIG. 1. Schematic diagram of the free-electron laser oscillator. (For more details see Ref. 6.)

Fig. 12. Madey's FEL oscillator configuration.

in reference [Murphy 1990]. It shows that the system can be unstable and exhibit exponential growth of the bunching factor $B$ and of the radiation amplitude $A$

$$
B=\sum_{n=1}^{3} b_{n} e^{i \lambda_{n} z}, \quad A=\sum_{n=1}^{3} a_{n} e^{i \lambda_{n} z}
$$

where the quantities $\lambda_{n}$ are a solution of the cubic equation

$$
\lambda^{3}-\delta \mu^{2}+2 \rho \lambda+1=0 .
$$

Exponential growth is obtained when the roots of the cubic equation are complex. For small values of the detuning the imaginary root is

$$
\operatorname{Im}(\mu)=1 / L_{G}=2 \sqrt{3} \rho / \lambda_{U} .
$$

The dependence of the imaginary part of the root on the detuning is shown in Figure 14. The behavior is quite different from that of the small gain case, shown in (18) and in Figure 11. For a long undulator, where the term with the exponential growth dominates, there is always growth of the radiation intensity as long as the detuning is smaller than a critical value of the order of 2 . For a short undulator the exponentially growing term does not dominate and all three roots contribute to the change in radiation intensity. This is the small signal case discussed before.

The curve in Figure 14 gives us another important characteristic of an FEL operating in the high gain regime, the gain bandwidth. The gain has a maximum for zero detuning and decreases when the detuning is larger or smaller than zero. Since the detuning is the relative change in the FEL frequency, from this curve we can obtain that the gain bandwidth is given once again by the FEL parameter. 


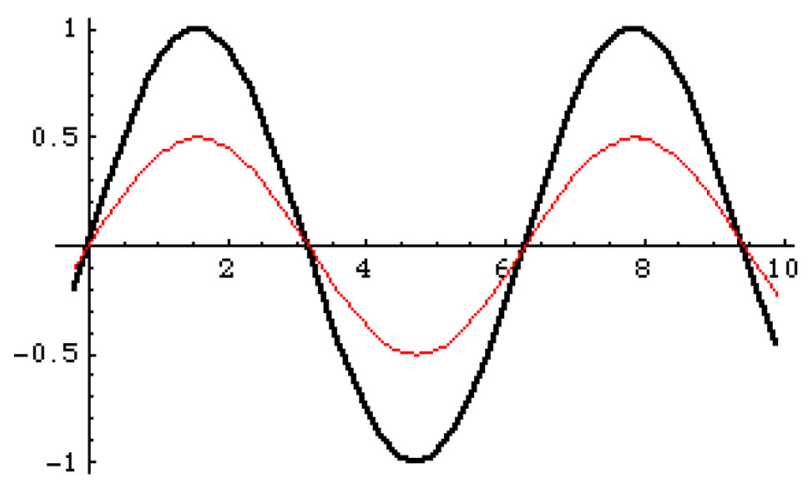

Fig. 13. The sinusoidal trajectories of two electrons in the undulator magnetic field. The black line is that of a low energy electron, and the red one that of a higher energy electron.

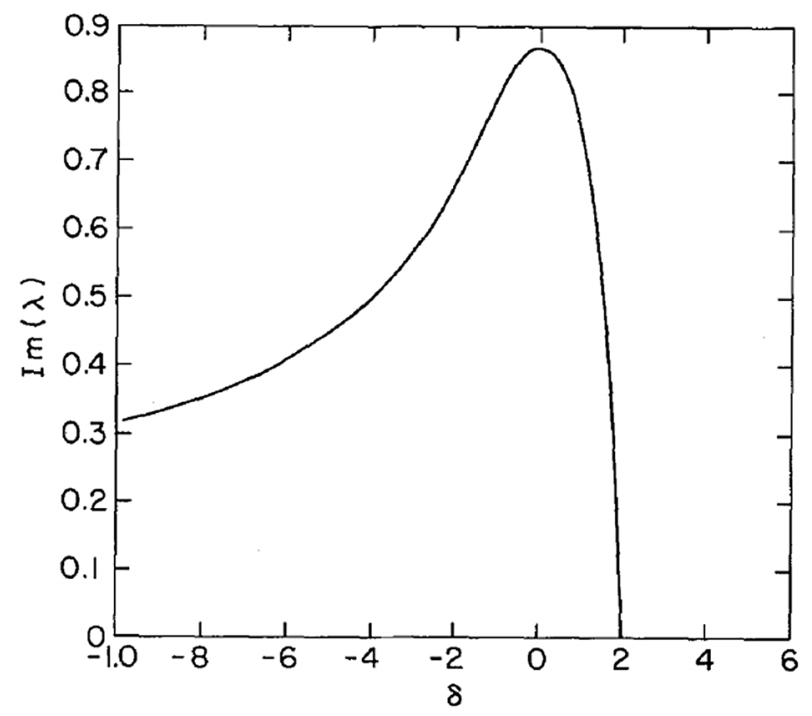

Fig. 14. The imaginary part of the root of the cubic equation as a function of the detuning. From reference [48].

The dynamics of the system can be better understood by looking at the electron phase space dynamics and bunching factor, as shown in Figure 15, and at evolution of the bunching factor and the radiation field intensity along the undulator axis as shown in Figures 16, 17.

These results are obtained integrating the $2 N_{e}$ equations describing the electron energy and phase change, together with two other equations describing the evolution of the phase and amplitude of the electromagnetic field. The calculation is done using normalized units: the length along the undulator is measured in units of the gain length (21) and the power is normalized to the saturation power (22). The initial condition is zero external electromagnetic field, and random electron distribution on the scale of the wavelength. This initial noise is what generates the spontaneous undulator radiation in the limit of a short undulator, short meaning that $L_{U}$, the undulator length, is smaller than the gain length. At the contrary, when $L_{U} \gg L_{G}$ the collective instability develops and the bunching and intensity grow exponentially 


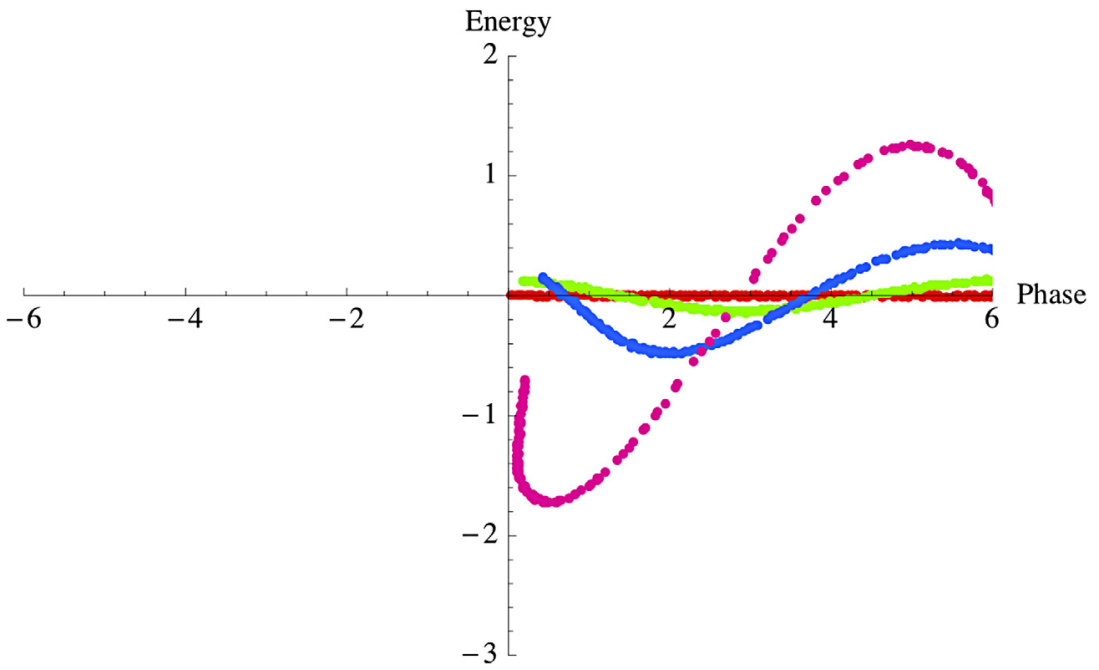

Fig. 15. Phase space evolution in the SASE high gain regime for a beam with small initial energy spread and random position distribution.

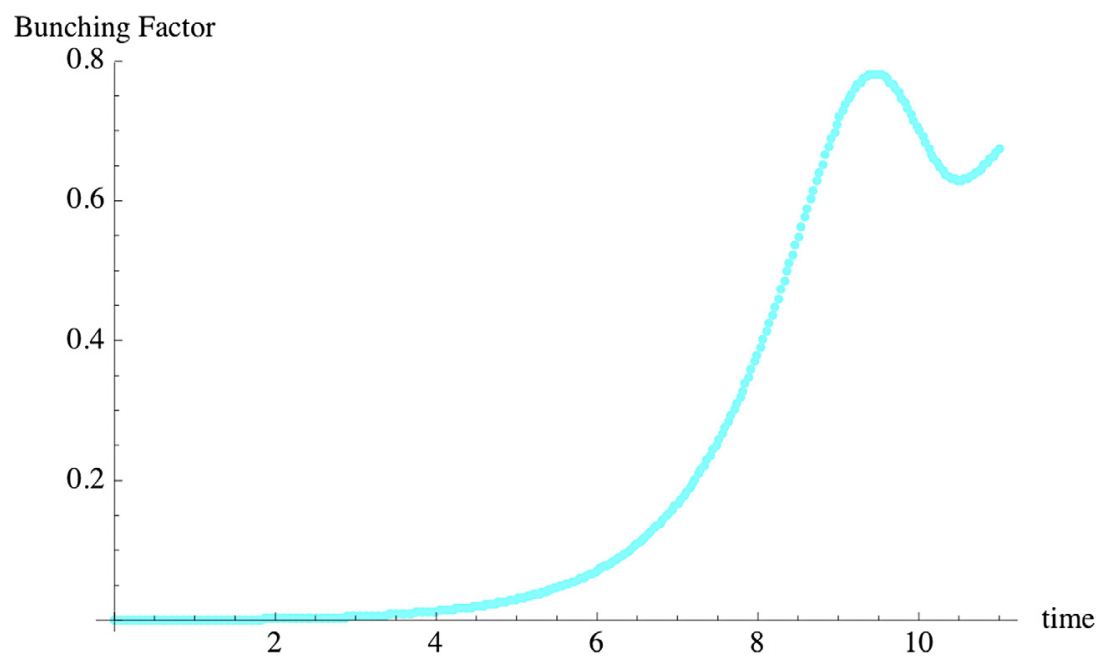

Fig. 16. Bunching factor growth along the undulator axis.

to saturation, which occurs in about ten gain lengths. This is the condition that we call Self Amplified Spontaneous Emission.

The plot of the intensity in logarithmic scale in Figure 18 shows clearly the exponential gain region, followed by saturation.

Typical values of $\rho$ for X-ray FELs are $10^{-3}-10^{-4}$. Using we can evaluate the number of coherent photons/electron in the radiation pulse at saturation: $N_{p h} \sim$ $\rho E / E_{p h}$. For $E_{p h}=10 \mathrm{keV}, E=15 \mathrm{GeV}, \rho=10^{-3}, N_{p h} \sim 10^{3}$, a gain of 5 orders of magnitude respect to the spontaneous radiation case.

The exponential growth occurs, and the previous results are valid, if three conditions are satisfied:

(a) the energy spread is smaller than the gain bandwidth,

$$
\sigma_{E}<\rho
$$




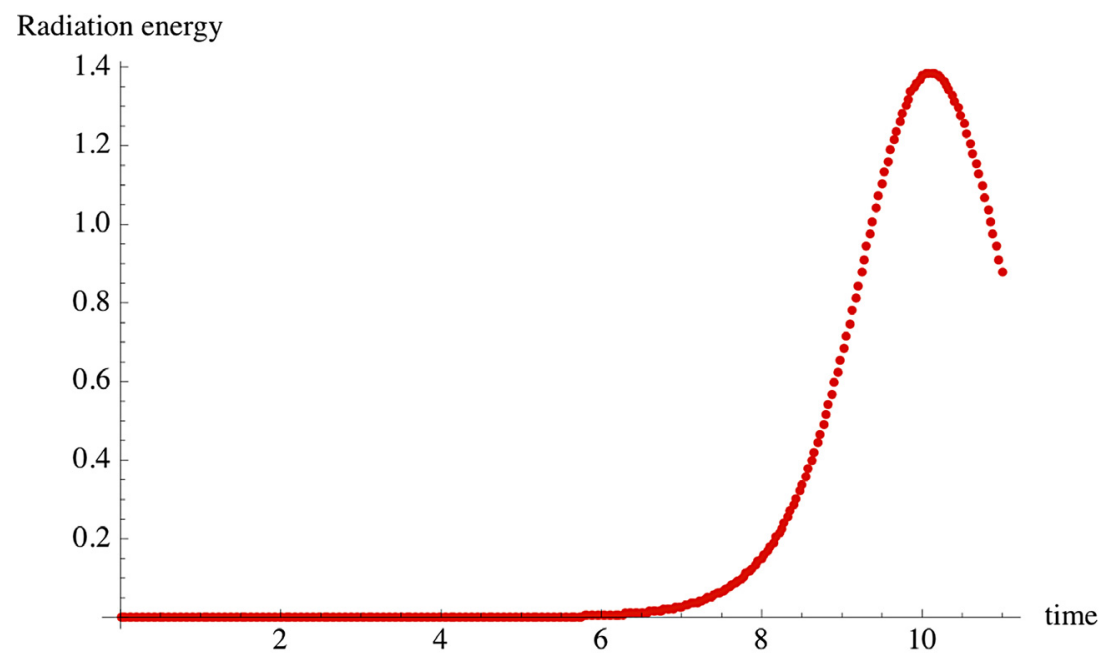

Fig. 17. Intensity growth along the undulator axis.

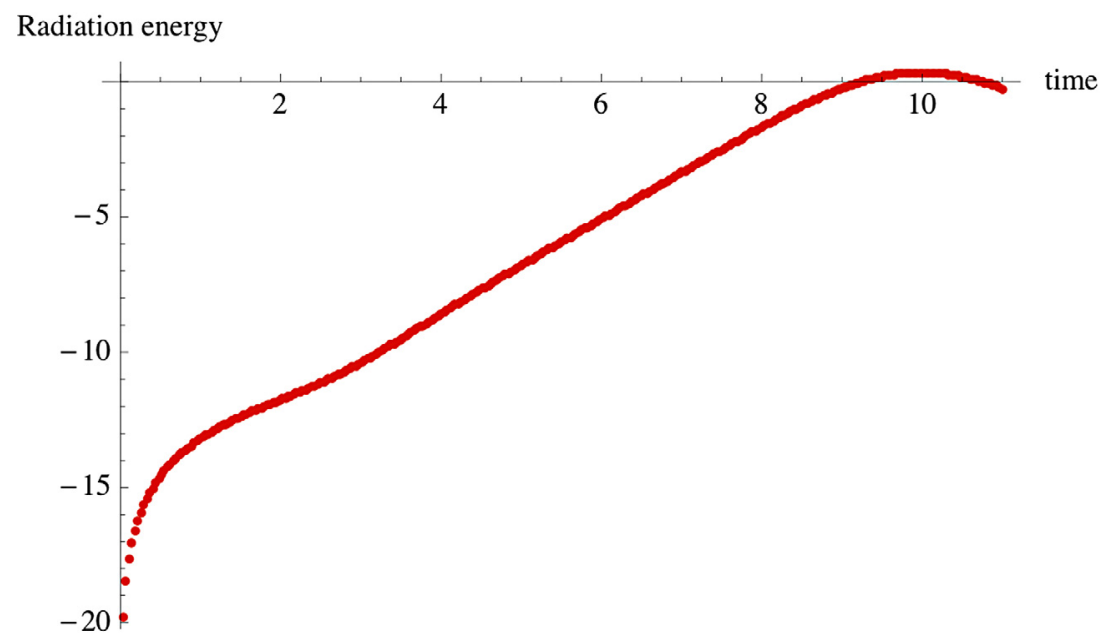

Fig. 18. The intensity growth along the undulator in logarithmic scale.

given by the FEL parameter; the beam must be cold, its energy spread small enough to avoid Landau damping of the instability;

(b) the radius and angular divergence of the electron and photon beams must be matched to provide a good overlap and interaction between photons and electrons;

(c) the diffraction losses from the radiation beam be smaller than the FEL gain, requiring that the radiation Rayleigh range be larger than the gain length, $Z_{R} / L_{G}>1$.

These conditions are very important. On one side they become more restrictive at shorter wavelength. On the other side they tell us what are the characteristics needed for the electron beam, and what kind of accelerator and electron source can be used.

As one can see from the definition of the FEL parameter, (20), the gain length is a function of the electron density. A larger electron density gives a larger value the beam plasma frequency and of the FEL parameter $\rho$. However the conditions just discussed put also limitations on the electron beam energy and momentum, or angular, spread. 
This means that for an FEL to work we must require that its six-dimensional phase space density, the number of electrons divided by the six-dimensional phase space volume, $V_{6}=x p_{x} y p_{y} z p_{z}$ be large. Here $z$ is the longitudinal coordinate along the undulator axis, and $x, y$ are the two transverse coordinates.

Neglecting collisions the electron-undulator-radiation field system is an Hamiltonian system and it follows from Liouville theorem [Liouville 1838] that its six-dimensional phase space density is an invariant. In most cases the three degrees of freedom are uncoupled and the three phase space areas $A_{x}=x p_{x}, A_{y}=y p_{y}$, $A_{z}=z p_{z}$ are separately conserved and are used, together with the charge or number of electrons, to characterize an electron beam.

In accelerator physics terminology the quantities that are used are called the normalized emittances, and are defined as the phase space area divided by $m c$, the electron rest mass times the light velocity. For the transverse case

$$
\varepsilon_{N, x}=\frac{A_{x}}{m c}=x \gamma \frac{d x}{c d t}=\gamma x \frac{d x}{d z}=\gamma x \theta
$$

where $\theta$ is the angle between the velocity in the $x$-direction and the $z$-axis. A similar evaluation can be made for the other directions. In most cases of interest to us the $x$ and $y$ normalized emittances are about equal. For a single electron the minimum transverse phase space area is determined by the uncertainty principle and is given by half the reduced Compton wavelength $\lambda_{C}=\hbar / m c=3.87 \times 10^{-13} \mathrm{~m}$.

Together with the normalized emittance another quantity commonly used is the emittance, the product of the transverse position and angular spread

$$
\varepsilon_{x}=\varepsilon_{N, x} / \gamma
$$

The emittance is only a beam invariant when the energy is not changing.

In the case of coherent photons, an ensemble of photons in the same state, the transverse phase space area is obtained again from the uncertainty principle as

$$
\varepsilon_{\text {photons }}=x \theta=\lambda / 4 \pi \text {. }
$$

Hence condition (b) discussed before can be written as

$$
\varepsilon_{N, T} / \gamma \approx \lambda / 4 \pi
$$

The most common unit for the emittance, or the normalized emittance is meters $\times$ radians. In this paper we will refer to the emittance in meters, omitting the radians, as is done in the most recent literature. Of course the numerical value does not change.

Condition (31), together with condition (27), put very stringent requirements on the electron beam needed to operate an FEL. As we already said, they become more restrictive at shorter wavelength. However, an analysis of the FEL scaling laws [Pellegrini 1988] in the high gain regime, using the scaling of the FEL parameter $\rho$ and of the conditions (a), (b), (c) for lasing, shows that gain length scales like the square root of the wavelength, a weak dependence favoring the extension to short wavelength respect to other types of lasers. This result is quite different from Madey's original scaling law and opens the way to X-ray FELs.

Generating electron beams satisfying these conditions and giving an FEL parameter value near to 0.001 has been the great challenge of X-ray FELs, as we will discuss in the next sections. If they can be satisfied, the X-ray FEL intensity will grow exponentially to reach saturation. The result is that, while in the spontaneous radiation case the total intensity is proportional to the number of electrons, $N_{e}$, in the high gain case the total intensity is proportional to a power of $N_{e}$ between $4 / 3$ and 2 . The number of electrons in a bunch is typically of the order of $10^{9}-10^{10}$, so the change 


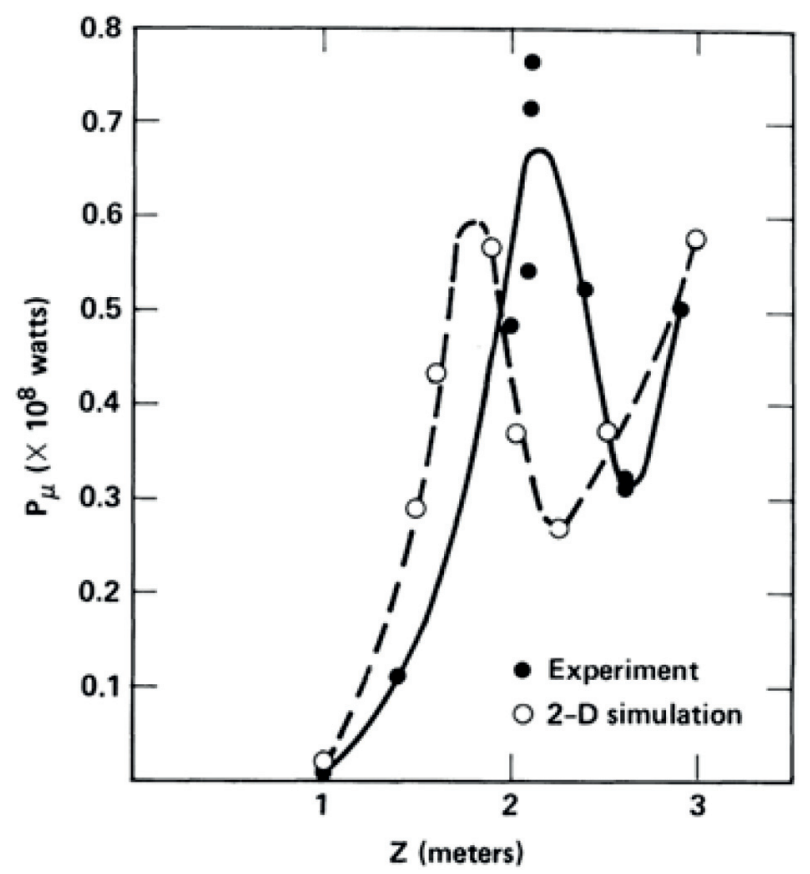

Fig. 19. Amplified signal output as a function of length along the undulator. The FEL operates at a frequency of $34.6 \mathrm{GHz}$, and the input signal, provided by a magnetron, is about 50 kW. From reference [Orzechowski 1985].

in intensity can be quite large. In fact the number of coherent photons emitted spontaneously by one electron going through an undulator is approximately given by the fine structure constant, or about $10^{-2}$. When a high gain FEL reaches saturation the number can be as large as $10^{3}-10^{4}$ [Pellegrini 2001].

The high gain exponential regime was first observed by a Lawrence Berkeley National Laboratory-Lawrence Livermore National Laboratory group, led by Donald Prosnitz, in 1985, at about $1 \mathrm{~cm}$ wavelength, with the radiation propagating in a waveguide, using a 3.3 to $3.8 \mathrm{MeV}$ electron beam from an induction linac [Orzechowski 1985]. The FEL reached saturation in about $1.3 \mathrm{~m}$ with an input signal of about $50 \mathrm{~kW}$, as shown in Figure 19. The FEL showed exponential gain when starting from spontaneous radiation noise, as shown in Figure 20.

The Berkeley-Livermore was very important. However, since the radiation was propagated in a wave-guide, it did not provide a full test of the diffraction effects and their consequences for an FEL. These effects are clearly more important at shorter wavelengths, when the radiation is propagating in vacuum. The three dimensional theory [Moore 1984; 1985; Scharlemann 1985; Kim 1986b; Krinsky 1987; Yu 1990] included diffraction losses, and showed the existence of refractive and gain guiding of the radiation. The guiding, first predicted theoretically by Moore [Moore 1984] and Scharlemann et al. [Scharlemann 1985], is of critical importance for lasing. Since diffraction is related to the electron beam radius, and the wavelength, lasing requires the gain in a Rayleigh range to be larger than the diffraction losses, as stated before in condition (c).

The last theoretical step needed was an understanding of the temporal structure of the radiation pulse in a SASE amplifier. The structure, and the related bandwidth, is due to the "slippage" effect. Because the photons move faster than the electrons, the radiation emitted by one electron moves ahead, slips, by one wavelength per undulator 


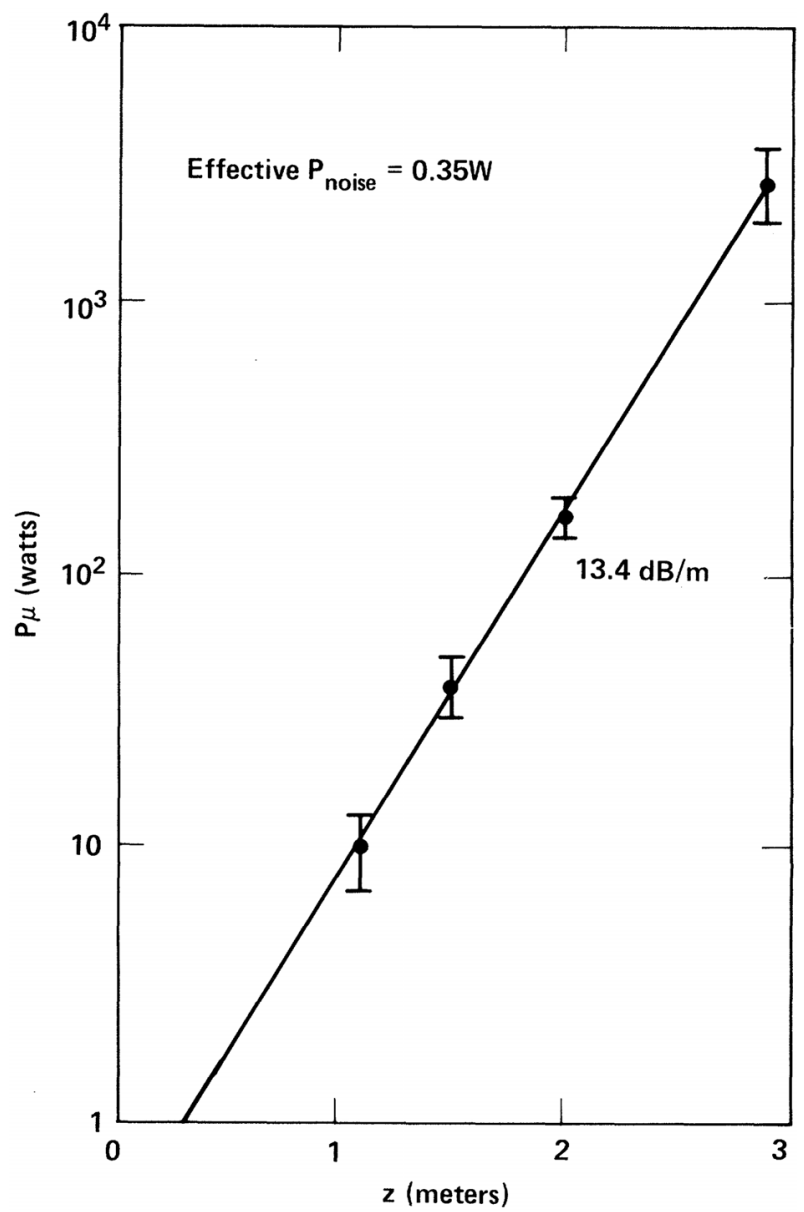

Fig. 20. Power as a function of undulator length in the Self Amplified Spontaneous Emission mode, starting from noise. From reference [Orzechowski 1985].

period, as one can see from (1). This means that for an undulator with $N_{U}$ periods, the radiation emitted by one electron only reaches electron ahead of it in a length $S=\lambda N_{U}$, the slippage length.

The analysis [Bonifacio 1994] of the effect of slippage for an electron bunch of finite length, when the slippage effect cannot be neglected, shows that the interaction between the electrons is only effective over a cooperation length, the slippage in one gain length. In a 1-dimensional model the cooperation length can be written, using (21), as

$$
L_{c}=\frac{\lambda}{2 \sqrt{3} \rho} .
$$

When starting from noise, and since the initial noise varies along the bunch length, the output radiation pulse consists of a series of spikes of random intensity separated by a distance proportional to the cooperation length. While in the case of spontaneous radiation the intensity along the pulse varies randomly in each wavelength, in the SASE case, at saturation, the interaction between electrons and their emitted radiation generates a number of spikes of random intensity and duration proportional to 

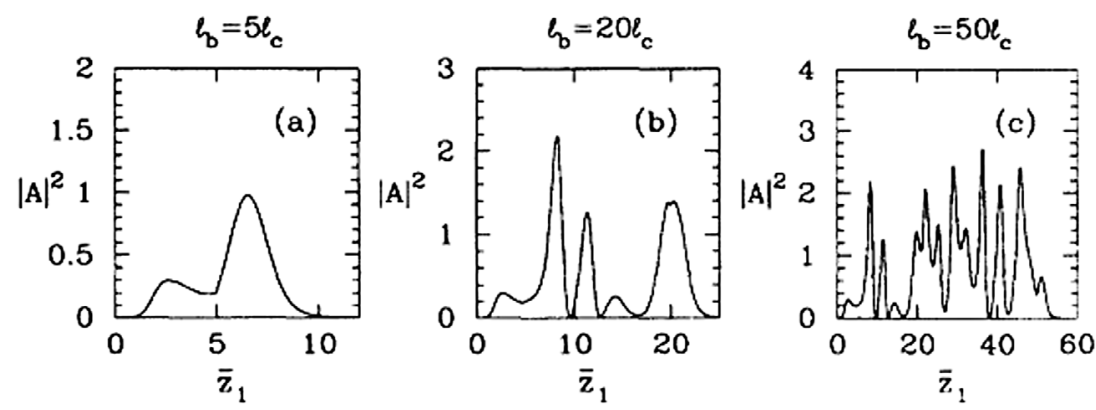

Fig. 21. Temporal structure of a SASE pulse near saturation as a function of the electron bunch length, from reference [Bonifacio 1994].

the cooperation length. The process and the output temporal structure of the X-ray SASE pulse is shown in Figures 21 and 22.

The number of spikes in a pulse is given by the ratio of the bunch length to the cooperation length. The intensity in each spike fluctuates from pulse to pulse and there is no correlation between the phases of different spikes. The statistical distribution of the total intensity, summed over all spikes, is given by a Gamma distribution function [Saldin 1998]. The line width, in a SASE FEL, is inversely proportional to the spike length, and not the bunch length. The width is of the order of the FEL parameter, $\rho$. Hence a SASE radiation pulse is not Fourier transform limited, except for the case of an electron bunch length shorter than the cooperation length, when a single spike is produced.

\section{Can we build an X-ray FEL?}

Following Madey's work, and while the FEL theory was being extended to the high gain regime, other infrared FEL oscillators were built and used for research in condensed matter, chemistry and biology, taking advantage of their tenability, the capability to operate over a large range of wavelength. The question of whether it would be possible to extend the FEL to the X-ray region was also being considered. The existence of the high gain regime offered the possibility of large gain and of reaching saturation in a single pass amplifier starting from spontaneous radiation, avoiding the need for a low-loss optical cavity, which was unavailable at X-ray wavelengths.

Claudio Pellegrini became very interested in the possibility of exploiting the high gain regime, studied in his work with Bonifacio and Lorenzo Narducci [Bonifacio 1984], to develop an X-ray free-electron laser. In 1985 James Murphy and Pellegrini analyzed the use of a SASE-FEL single-pass amplifier starting from noise, inserted in a storage ring, to produce soft X-rays [Murphy 1985a; 1985b]. The analysis used the electron beam from a storage ring, the accelerator giving the greatest beam density obtainable at that time. Detailed knowledge of the beam properties and limitations in a storage rings had been obtained in the two previous decades from the work done to build high luminosity electron-positron colliders. The beam current, energy spread and phase space density were included in the analysis and evaluated including all known effects. The main limitation to the FEL gain comes from the energy spread, which is larger than 0.001 at a peak current of a few hundreds Amperes, while the normalized beam emittance is a few times $10^{-6} \mathrm{~m}$. The result was that, when using a storage ring, the shortest FEL wavelength achievable is about $50 \mathrm{~nm}$, a conclusion still valid today. Similar results were obtained in other analyses of storage ring FELs [Cornacchia 1986; Gover 1986]. 
Avg. Field Power vs. Z

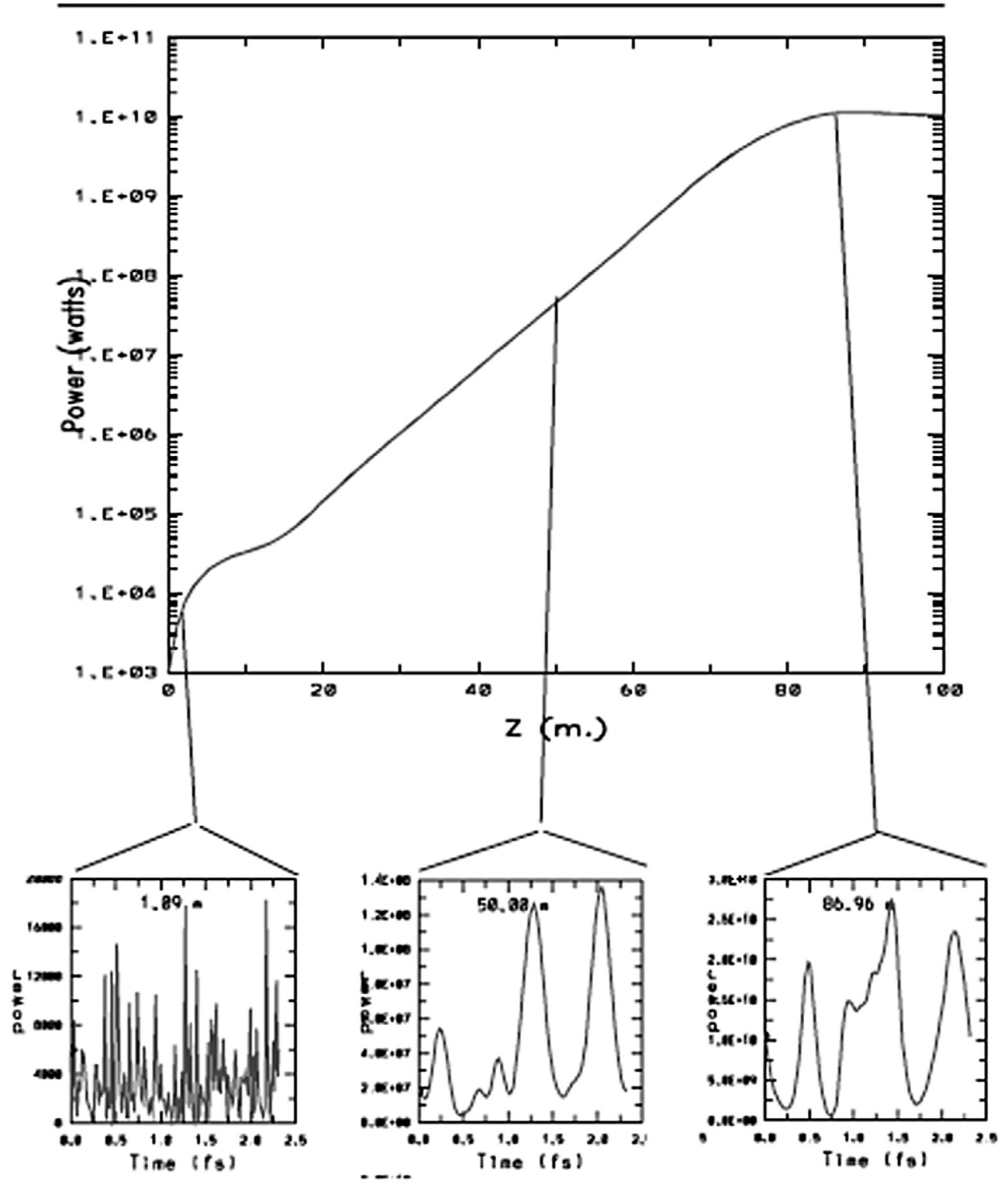

Fig. 22. The intensity and temporal structure evolution of a SASE radiation pulse for the LCLS case [Cornacchia 1998]. The random intensity fluctuation for a short undulator is that of the spontaneous radiation, changing on the scale of the radiation wavelength. Because of the FEL interaction the initial spikes coalesce in longer spikes, of length proportional to the cooperation length.

An alternative to storage rings was needed to reach shorter wavelengths, and Pellegrini turned his attention to another high-energy accelerator, the linear accelerator [Pellegrini 1988]. In contrast to the storage ring case, the analysis showed that using a linear accelerator together with a new photo-injector electron source devel5 oped at Los Alamos [Fraser 1986; 1987] by a group led by Richard Sheffield, it was 6 possible to reach nanometer to Angstrom wavelengths. The new type of gun generated 
an electron phase space density larger than any other gun existing at that time by at least one order of magnitude. The possibility was further enhanced by Bruce Carlsten's idea of emittance compensation [Carlsten 1989] to generate the desired electron beam characteristics, a gun peak current of about $100 \mathrm{~A}$ and a transverse normalized emittance of a few times $10^{-6} \mathrm{~m}$, compared to values of $10^{-5}$ to $10^{-4}$ common at that time using thermionic guns. As an example the normalized emittance reported for the SLAC linac in the Blue Book [Neal 1967] is about $2 \times 10^{-3} \mathrm{~m}$ at a peak current of $3.8 \mathrm{~mA}$, for away from what is needed for an X-ray FEL.

This work by the Los Alamos group attracted the attention of Robert Palmer and Pellegrini, who, in 1987 had created at Brookhaven National Laboratory the Center for Accelerator Physics, with a program of research in laser and other advanced methods of acceleration, and FELs. The Center was supported by the Brookhaven laboratory and by the DOE program for advanced accelerator physics and technology directed by David Sutter, within the Office of High-Energy Physics. The central instrument of the Center was a $60 \mathrm{MeV}$ linac. A high brightness electron source generating an electron beam with high current and high phase space density was needed to carry out this program. After extensive discussions, Palmer and Pellegrini decided to base the program on an S-band version, at $2.8 \mathrm{GHz}$, of the Los Alamos photo-injector operating in L-band at the frequency of $1.3 \mathrm{GHz}$.

Batchelor [Batchelor 1988; Qiu 1996] and McDonald [McDonald 1988] led the photoinjector design effort. While the gun was being developed, the exploration of the possibility of building an X-ray FEL continued both at Brookhaven and at UCLA, where Pellegrini moved in 1989. Here he continued his work on FELs with the support of DOE and additional funding from UCLA. To provide an experimental test of the high gain SASE theory he set-up a new laboratory, having as its main instrument a low energy, $20 \mathrm{MeV}$, linac. The electron source was again an S-band photoinjector, based on the Brookhaven design. This critical component was developed and built under the direction of James Rosenzweig, who had just joined UCLA as an Assistant Professor.

In 1990 Palmer and Gallardo organized a workshop at Sag Harbor to explore again the feasibility of a $1 \AA$ FEL [Gallardo 1990]. Two questions that were asked to the participants: "What are the prospects for a $1 \AA$ Free-Electron Laser? Can we obtain electron sources bright enough to get down to the $1 \AA$ region"? In the workshop Pellegrini discussed the status of the research on short wavelength FELs, including an analysis of the scaling laws, and showing that the FEL parameters, and thus the gain length, scales like the square root of the wavelength, a weak and favorable dependence [Pellegrini 1990]. His conclusions were: "The FEL in the SASE regime offers an attractive route to an X-ray laser. To make this laser a reality it is necessary to solve many problems; produce electron beams with very high quality and refine the understanding of the physics of FELs. We also need to produce long, shortperiod undulators with good field quality. To reach these goals we need an extensive experimental and theoretical effort on electron guns, accelerators and FELs with a number of intermediate steps that will take us from the present region of $240 \mathrm{~nm}$, and $1 \mathrm{~W}$ to $0.1-1 \mathrm{~nm}$ and $1 \mathrm{GW}$ ".

The physics and status of RF photoinjectors, which were identified as the more promising system to reach the desired goals of beam transverse emittance, where discussed at the workshop by Kim [Kim 1990]. In particular it was recognized that the peak current and energy spread obtainable from RF photoinjectors, according to the Los Alamos results at L band and the simulations for the S-band case, were much superior to those obtainable in an electron storage ring, and satisfied the requirements for an X-ray SASE-FEL. Kim also presented an analysis of the physics and technology of RF photoinjectors, discussing methods to reduce the emittance blow-up due to space-charge forces and RF curvature effects. 
Table 1. Assumed electron beam parameters in reference [Pellegrini 1992].

\begin{tabular}{lc}
\hline Normalized rms emittance, $\mathrm{m}$ & $2.5 \times 10^{-6}$ \\
\hline Peak current, A & 2000 \\
Pulse duration, rms, ps & 0.16 \\
Relative energy spread, rms, \% & 0.04 \\
\hline
\end{tabular}

Table 2. Parameters for a $4 \mathrm{~nm}$ FEL, from reference [Pellegrini 1992].

\begin{tabular}{lc}
\hline Electron energy, GeV & 6 \\
\hline Undulator period, cm & 6.8 \\
Undulator field, T & 0.63 \\
Betatron Wavelength, m & 10 \\
FEL Parameter & 0.002 \\
Gain length, m & 3.2 \\
Raleigh range, m & 1.6 \\
Pulse length, rms, ps & 0.16 \\
Undulator saturation length, m & 34 \\
FEL Power, GW & 24 \\
\hline
\end{tabular}

At the same time, the work done at SLAC, by Bane, Seeman, Raubenheimer and others, to develop the SLAC linear electron-positron collider (SLC), demonstrated the feasibility of accelerating and compressing electron beams, to enhance the peak current to a few hundred amperes, without increasing the emittance [Bane 1987; Seeman 1991a; 1991b; Raubenheimer 1997].

These theoretical, experimental and technological developments, hinted at the possibility of an X-ray FEL. Max Cornacchia and Herman Winick, from the Stanford Synchrotron Radiation Laboratory (SSRL) at SLAC, organized in 1992 a Workshop on Fourth Generation Light Sources [Cornacchia 1992], which provided a very good opportunity to review and discuss the progress in many areas of synchrotron radiation generation and the possibility of a significant step in their brightness and other characteristics. SSRL had been a pioneer in the use of an electron storage ring, the SPEAR electron-positron collider at SLAC, to generate and use synchrotron radiation in physics and chemistry. Cornacchia and Winick considered that the time was right to look beyond existing capabilities. In fact, at the workshop Pellegrini presented a seminal proposal to build a 0.1 to $1 \mathrm{~nm}$ SASE-FEL using the SLAC linac and a photoinjector [Pellegrini 1992].

Madey and coworkers developed the FEL on the Stanford University campus in the 1970s. After that time other laboratories, like Los Alamos and Livermore National Laboratories, industries and universities had started high average power FELs programs, mostly funded by the Strategic Defense Initiative program. Pellegrini's group, first at Brookhaven National Laboratory and later at UCLA, was one of the few to concentrate its work on short wavelength, nanometer or sub-nanometers, FELs, for research use in physics, chemistry and biology, with funding from the High Energy Physics office of DOE for the development of high brightness electron beams. SLAC had remained out of the FEL research, also because most funds for this field came from military programs. There was however at SLAC a high energy linac, and the knowledge of beam physics relevant to the acceleration and compression of small emittance beams. These assets gave SLAC a unique position for the development of an X-ray FEL, and motivated Pellegrini's choice to propose to build it there.

The proposed parameters for the electron beam and a $4 \mathrm{~nm}$ FEL are shown in Tables 1 and 2, from [Pellegrini 1992]. The emittance assumed for the electron gun was the major issue for the extension to $0.1 \mathrm{~nm}$, as stated in Pellegrini's 
Table 3. The $4 \mathrm{~nm}$ FEL considered in the initial work of the design group.

\begin{tabular}{lc}
\hline Electron energy, GeV & 3.5 \\
\hline Normalized rms emittance, m & $3 \times 10^{-6}$ \\
Peak current, A & 2500 \\
Energy spread, rms, \% & 0.07 \\
Undulator period, cm & 5 \\
Undulator field, T & 0.8 \\
FEL Parameter, & 0.0008 \\
Field gain length, m & 5 \\
Pulse length, rms, ps & 0.16 \\
Undulator saturation length, m & 48 \\
FEL peak power, GW & 10 \\
\hline
\end{tabular}

conclusions: "We have shown that using existing electron gun technology and the SLAC linac one can build today a FEL in the water window, at about $4 \mathrm{~nm}$. An improvement in the gun emittance by a factor of three for the same longitudinal brilliance, would allow the extension of the system to $0.1 \mathrm{~nm}$. In both cases the radiation brightness far exceeds any other source existing or under construction, and would open a completely new region of experimentation."

It was generally acknowledged that the proposal was at the frontier of accelerator physics and technology, and many scientists considered the requirements on the electron beam, together with the needed undulator magnetic field tolerances and electron beam alignment, as practically impossible to achieve. It was also well understood that extrapolating the SASE theory from the centimeter wavelength of the Livermore experiment [Orzechowski 1985], or from the low gain infrared oscillators, to the Angstrom region, was risky. However Herman Winick, Arthur Bienenstock, then the director of the synchrotron radiation laboratory at SLAC, Burton Richter, then the director of SLAC, and some other scientists saw the great potential impact of an X-ray FEL on all field of atomic and molecular science. Winick took the initiative to form a study group comprised mostly of SLAC scientists, together with Pellegrini and some other people, to develop the concept, analyze the critical R\&D issues, and prepare an initial design of an X-ray FEL. The first meeting of the study group was held very soon after the workshop, on March 18, 1992. The memorandum with the minutes of the first meeting and the study group members, are shown in Figures 23 and 24.

The study group held regular meetings, mostly at SLAC and some at UCLA. Initially it concentrated its efforts on a 2 to $4 \mathrm{~nm}$ FEL, the so-called water window wavelength range, considered to be a safer initial step toward the Angstrom region. The work was summarized in a paper presented at the August 1992 International FEL conference, held in Kobe, Japan [Pellegrini 1993]. The FEL main characteristics presented in this paper are given in Table 3.

In April it was considered to submit a proposal for a 2 to $4 \mathrm{~nm}$ FEL to the US Department of Energy for construction starting in 1995, and development work to be done between 1992 and 1995. The name LCLS, introduced by Winick, appears for the first time in a memorandum dated June 13, 1992.

A workshop on "Scientific applications of short wavelengths coherent light sources", chaired by William Spicer and co-chaired by John Arthur and Herman Winick, was also organized for October 1992. One of the main applications considered for the water window FEL was X-ray microscopy of biological cells, a field that had seen a considerable development in the 1980s [Jacobsen 1998]. However many scientists participating in the workshop expressed a strong concern about the practicality of the X-ray FEL. The main concern was that the very high X-ray intensity would 
Notes on linac-based FEL meeting of March 18, 1992

Subject: Notes on linac-based FEL meeting of March 18, 1992

From: WINICK\%SSRL01@SSRL.SLAC.STANFORD.EDU

Date: Wed, 25 Mar 1992 19:13:33 -0700 (PDT)

CC: WINICK@SSRL.SLAC.STANFORD.EDU

March 25, 1992

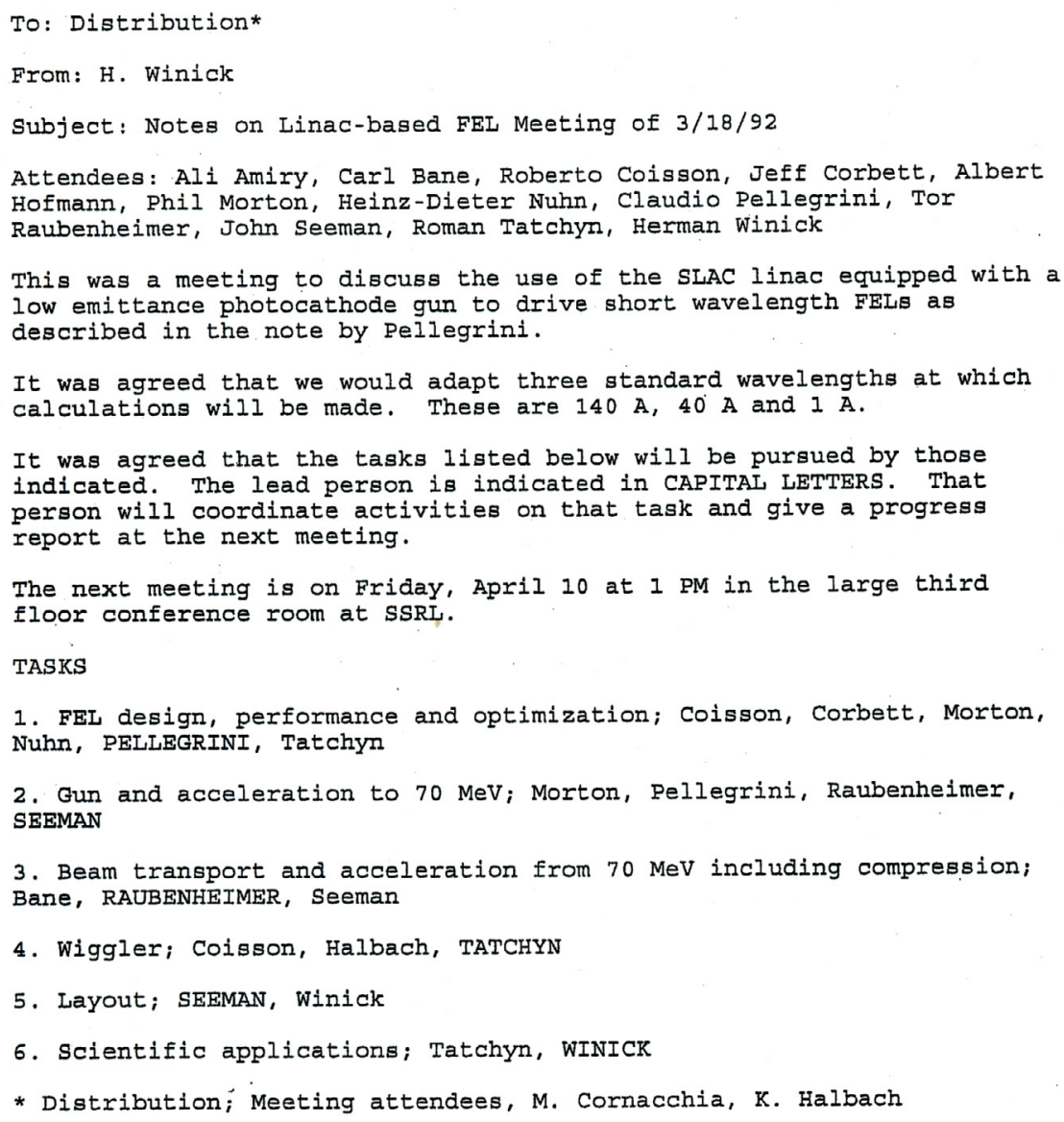

Fig. 23. Minutes of the first meeting of the X-ray FEL study group.

blow-up any sample on the beam path, making it impossible to obtain useful data, contrary to what happens in a storage ring based synchrotron radiation source, were the intensity of each pulse is low, the sample can survive essentially unchanged, and data from many exposure can be accumulated at the very high repetition rate of the ring, in the $\mathrm{MHz}$ range.

The combined skepticism of many X-ray and FEL scientists on the practicality and feasibility of the X-ray FEL project was a high hurdle to overcome, and made very difficult to obtain support from funding agencies, as we will see later in this paper.

In any case the study group continued its work and later on, in November 20, 21 of the same year, it presented its results to a technical review committee, chaired by 


\section{SHORT WAVELENGTH FELS AT SLAC - STUDY GROUP}

\section{SOURCE}

Karl Bane
Jeff Corbett
Max Cornacchia
Klaus Halbach (LBL)
Albert Hofmann
Kwang-je Kim (LBL)
Phil Morton
Heinz-Dieter Nuhn
Claudio Pellegrini (UCLA)
Tor Raubenheimer
John Seeman
Roman Tatchyn
Herman Winick

SCIENTIEIC CASE

\author{
Art Bienenstock \\ Keith Hodgson \\ Janos Kirz (SUNY-Stony Brook) \\ Piero Pianetta \\ Steve Rothman (UCSF) \\ Brian Stephenson (IBM)
}

Fig. 24. The list of the first members of the study group.

Ilan Ben Zvi. The other committee members were Joseph Bisognano, Luis Elias, John Goldstein, Brian Newnam, Kem Robinson, Andrew Sessler and Richard Sheffield. The review committee was impressed with the possibility of making a large leap down in FEL wavelength. Their main conclusion was that there is no physical principle saying that the device would not work. However there where a few uncertainties on some beam parameters, and R\&D was needed and recommended in some critical areas, in particular the electron source emittance and longitudinal pulse compression, defining the electron density and peak current, and the beam alignment in the undulator system and strategy, since the tolerances were estimated to be of the order of $20 \mu \mathrm{m}$.

In August 1993 SLAC, Brookhaven National Laboratory (BNL) and UCLA formed a collaboration to further develop the electron gun, using a common photoinjector design, and reduce the emittance by a factor of two to three, as required to reach the $1 \AA$ wavelength.

In the mean time the construction of a $4.5 \mathrm{MeV}$ photoinjector gun, based on the Brookhaven design, was completed at UCLA and first measurements of the emittance were reported in 1994 [Hartemann 1994].

As part of the collaboration Winick proposed, successfully, to build a Gun Test Facility (GTF) at SLAC, in addition to the work already underway at Brookhaven and UCLA. The GTF was located in an existing vault at SLAC, next to the electron storage ring SPEAR, where electrical and other utilities, radiation shielding and radio-frequency power were already available. The accelerator was a $3 \mathrm{~m}$ S-band linac section. The test facility was built and commissioned by John Schmerge and James Weaver. David Reis and David Meyerhofer, from the University of Rochester, developed the photoinjector laser. Roger Miller and Dennis Palmer developed an improved gun design, called the Next Generation Photoinjector [Palmer 1998]. A drawing of the new gun is shown in Figure 25. The BNL-SLAC-UCLA collaboration completed the design and fabricated four copies of this gun. Four of these guns were machined at UCLA and then brazed and cold tested at SLAC. High power testing proceeded 


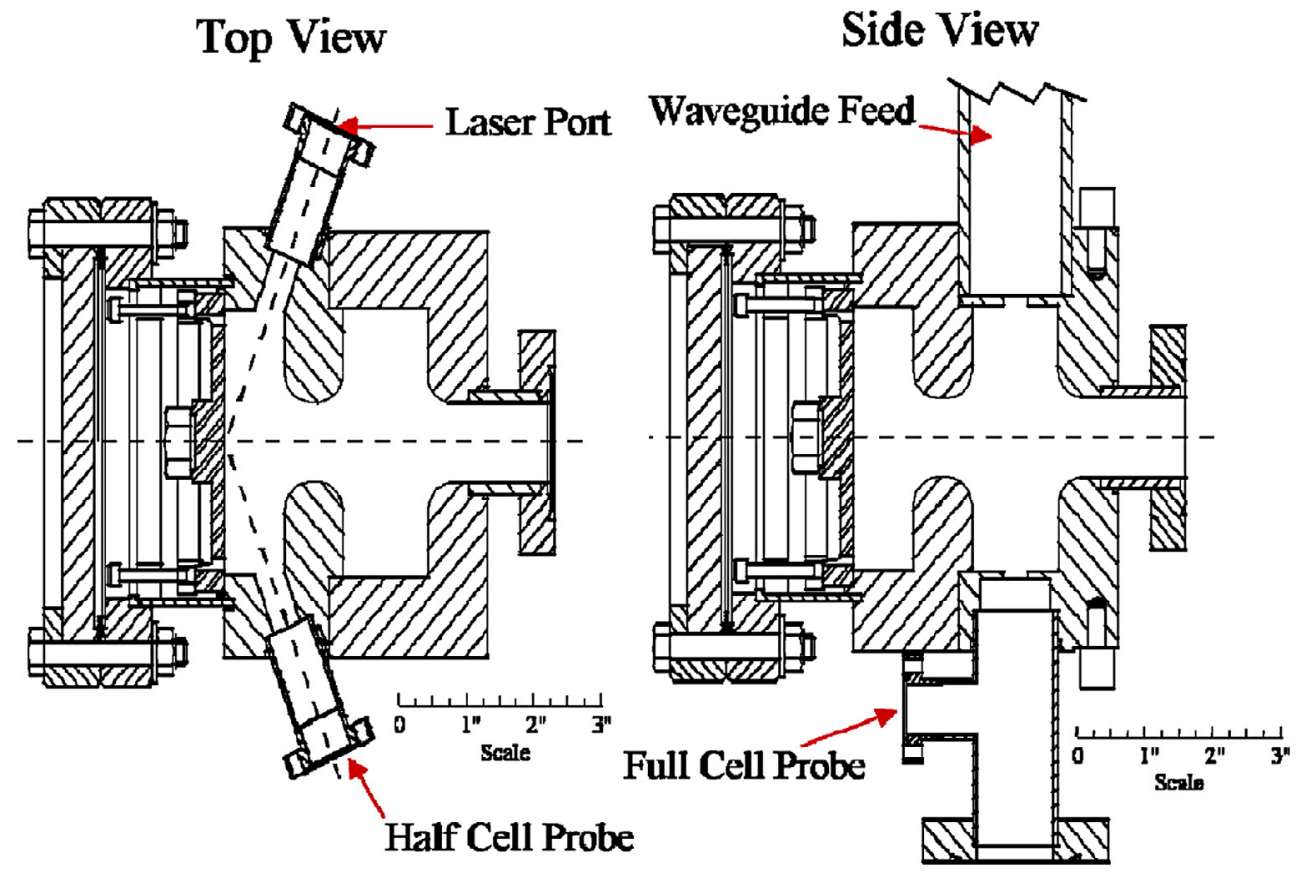

Fig. 25. Drawing of the third generation photo-injectors developed by the BNL-SLACUCLA collaboration.

at the GTF in 1996 and 1997. Two of the guns were used at Brookhaven National Laboratory, a third went to UCLA and the fourth was characterized in detail at the GTF. Measurements of the normalized emittance, of about $10^{-6} \mathrm{~m}$, with this gun were reported in 1997 [Palmer 1997].

The next generation gun generated beams with a smaller emittance than the original S-band Brookhaven gun. It was used in the experiments, discussed in the next section, demonstrating the SASE High Gain FEL theory, and thus gave an important contribution to the development of X-ray FELs. Even if it did not achieve the beam quality needed for $1 \AA$, it was also instrumental in showing how to design and build the LCLS electron gun, which reached the desired emittance values, and allowed to successfully generate coherent hard X-ray photons. The results obtained with the GTF gun and the development of the LCLS gun are reviewed and summarized in reference [Dowell 2008].

The work of the design group for an FEL conceived to operate in the water window (2-4 nm) or shorter wavelengths was presented at several conferences and workshops [Pellegrini 1994; Winick 1994; Travish 1995].

The response of the US Department of Energy to the LCLS collaboration request of funding for the development of the project was to ask the USA National Research Council (NRC) to review the status of FELs and recommend a course of action. The NRC formed a "Committee on Free Electron Lasers and Other Advanced Coherent Light" that issued his report in 1994 and gave a negative recommendation for the development of X-ray FELs [Levy 1994].

The report recommendations were:

1. Scientific opportunities and the use of coherence in the X-ray spectral region should be explored initially by the use of existing and planned synchrotron sources. 
2. The research and development necessary for the possible construction of an X-ray free electron laser should be supported. The goals of this research and development should be improving the technology and lowering the cost.

3. Research and development on other advanced coherent X-ray sources should continue to be supported. One of the goals of this research and development should be the production of devices of appropriate size and cost to be useful for scientific research on a departmental or individual-investigator scale.

4. Construction of an X-ray free electron laser user facility should not be undertaken at the present time.

Another workshop on "Scientific Applications of Coherent X-rays" was organized in 1994, chaired by Arthur, Materlik and Winick, and it provided much needed scientific support for the an X-ray FEL at SLAC [Arthur 1994]. The executive summary, prepared by Birgenau, Fadley and Materlik, states: "Finally a comment on the economic aspects of the proposed project is appropriate. The chance to build this source at SLAC seems to be unique worldwide. A section of the operational linac and existing buildings can be used. This would probably reduce the capital investment by about $80 \%$ of the total cost as compared to building such a source from scratch. Machine experts have pointed out that additional R\&D efforts would be needed to get from the original 40 A project study (feasible with present electron gun and linac technology) to a $1.5 \AA$, LCLS; however, no insurmountable problems are foreseen with this. This workshop and its predecessor have thus shown that a wide variety of new and exciting experimental possibilities in physics, chemistry, materials science, and biology would be opened up by an LCLS. Such an X-ray laser should in fact lead to the same sort of revolutionary developments in X-ray studies of matter that was produced in optical studies by the introduction of the visible/UV laser."

An additional important development was the beginning of the interest in X-ray FELs at the DESY laboratory in Hamburg. Bjorn Wiik was on sabbatical at SLAC during 1992. During his stay at SLAC he was informed by Herman Winick of the work being done on LCLS, and was present at the first LCLS review in November 1992. After the review he went back to DESY and he became the laboratory director in January 1993. Later Wiik and scientists in the DESY synchrotron radiation laboratory (HASYLAB), like Gerhard Materlik, became interested in the possibility of building an X-ray FEL as part of the electron-positron linear collider project, TESLA, being developed at DESY, using superconducting linear accelerators. A 1 GeV superconducting linac TESLA Test Facility (TTF) was being built at DESY to develop superconducting radio frequency technology for TESLA [Edwards 1995] and could be also used for a soft X-ray FEL. Joerg Rossbach was asked to lead the design and development of a VUV FEL [TESLA 1995; Rossbach 1996], a project that led eventually to the FLASH Soft X-ray FEL.

Even if the National Research Council report on FELs did not support funding the development of an X-ray FEL, the work by the Winick study group continued, with support from SSRL/SLAC, UCLA and some other groups. The interest moved back from the water window FEL to shorter wavelength, about $1.5 \AA$, that attracted more support from X-ray scientists. The study group was followed near the end of 1995 by a design group, under the direction of Cornacchia, that produced a full conceptual design report of the system [Cornacchia 1998], and extended the operating wavelength to $0.15-1.5 \mathrm{~nm}$.

The conceptual design report study group included scientists from Lawrence Livermore Laboratory, Los Alamos National Laboratory, UCLA, Lawrence Berkeley National Laboratory, the European Synchrotron Radiation Facility, the University of Rochester, the University of Milan, and DESY. The full list of names is found in [Cornacchia 1998]. 
A committee, chaired by Joseph Bisognano of Lawrence Berkeley laboratory, and including Gerd Materlik and Joachim Pflueger from DESY, reviewed the conceptual design report in November 1997. The committees report was complimentary of the way the study was making progress, and provided useful comments and suggestions for further work.

A new panel to evaluate the status and future developments of synchrotron radiation facilities in the United States was called by the Basic Energy Science division of the US Department of Energy in 1997. The panel was chaired by Robert Birgenau, and gave its recommendation in January 1998 [Birgenau 1997]. The work done by Cornacchia's LCLS design team and that of the groups working on photoinjector gun development and other aspects of the electron beam dynamics, was important for the panel deliberations. For the first time the report included among the top priorities the funding of R\&D for LCLS. It also discussed in the final recommendations the priorities for the R\&D effort: "The R\&D plan should emphasize small experiments establishing the capability of reaching the electron beam quality necessary for an X-ray FEL. The laser-driven photocathode gun, electron pulse compression in the accelerator, electron pulse transport in the undulator, and SASE experiments at longer wavelengths are all-important technologies. Building a prototype FEL at longer wavelengths is not as important as understanding the basic physics for the $1 \AA$ FEL. The decision for a start date on an X-ray FEL would depend on the success of these small experiments. The research should be a national effort involving universities and national laboratories. The actual distribution of research funds and schedule should be determined by another panel made up of potential users, accelerator and FEL physicists."

\section{Early SASE experiments}

At this stage, the theoretical and design work needed more experimental data verifying the SASE-FEL theory in all its aspects, including diffraction effects and the temporal spiky structure with the associated intensity fluctuations, and more data on beam brightness obtainable from photo-injectors. The work done by the LCLS collaboration had raised much interest in SASE-FELs, and other groups outside the collaboration started experimental work in this area. Two more experiments in the microwave region [Kirkpatrick 1989; Lefreve 1999] confirmed the initial Berkeley-Livermore results [Orzechowski 1985] on exponential gain. However it was important to demonstrate high gain at shorter wavelengths and for radiation propagating in vacuum instead of a waveguide. The first evidence of high gain starting from noise in the infrared spectral regions was obtained at the Laboratoire de l'Acceleratuer Lineaire at Orsay [Prazeres 1997] and at Brookhaven National Laboratory [Babzien 1998]. The first detailed measurements of the high-gain SASE regime, including the statistical intensity fluctuations characterizing the process, were made in the infrared region of the spectrum at UCLA by a group formed by scientists at UCLA and at the Kurchatov Institute in Moscow, led by Pellegrini, Rosenzweig and Alexander Varfolomeev, demonstrating exponential gain over four gain lengths, in a 60-cm-long undulator, at a wavelength of $16 \mu \mathrm{m}$ [Hogan 1998]. The undulator used in this experiment is shown in Figure 26.

The measured intensity and intensity fluctuations are shown in Figures 27 and 28. In this experiment, done with a fixed undulator length, the intensity is measured as function of the electron charge and current, thus changing the gain length, while keeping the undulator length fixed. The fluctuations are measured by selecting events with a well-defined and restricted range of electron bunch parameters. The experiment utilized a $20 \mathrm{MeV}$ linac built at UCLA and an undulator magnet built by Alexander Varfolomeev's group. [Varfolomeev 1995]. 


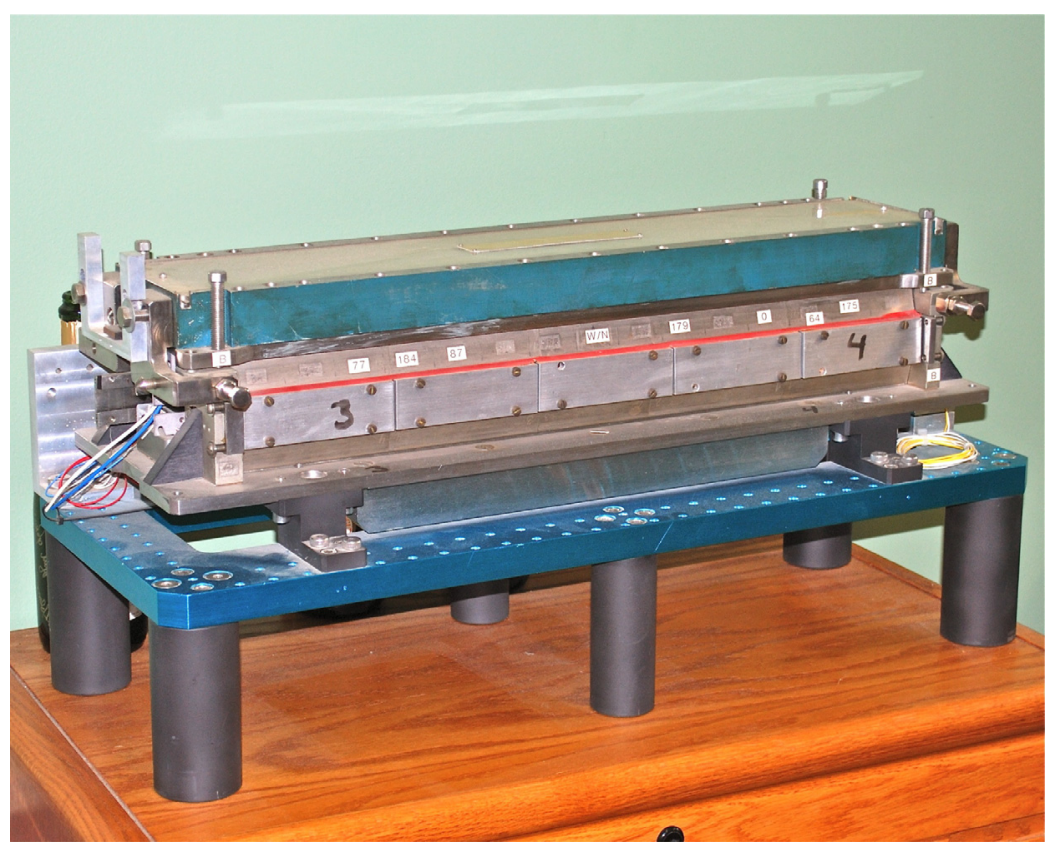

Fig. 26. The $60 \mathrm{~cm}$ long, $K=1$, period $1.5 \mathrm{~cm}$, undulator used for the first UCLA SASE experiment. The undulator was built at the Kurchatov Institute in Moscow, by the group led by Alexander Varfolomeev.

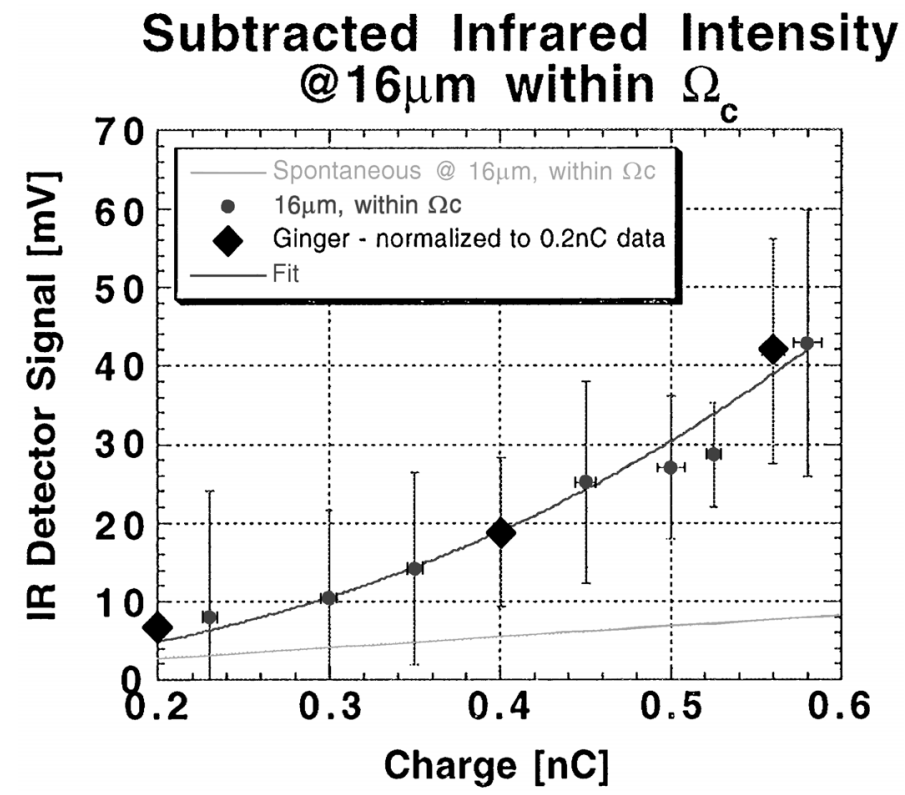

Fig. 27. First harmonic, coherent IR intensity versus charge. The vertical bars are the standard deviation for the intensity fluctuations. For comparison the effect of beam charge and radius uncertainties is $9 \%$ or a standard deviation of $4 \mathrm{mV}$ at $0.56 \mathrm{nC}$. The straight line is the calculated spontaneous emission intensity while the curved line is a fit to the data $I=1.85 Q \exp \left(4.4 Q^{1 / 3}\right)$. The three diamonds at $0.2,0.4,0.56 \mathrm{nC}$ are the normalized results of simulations with the code Ginger. 


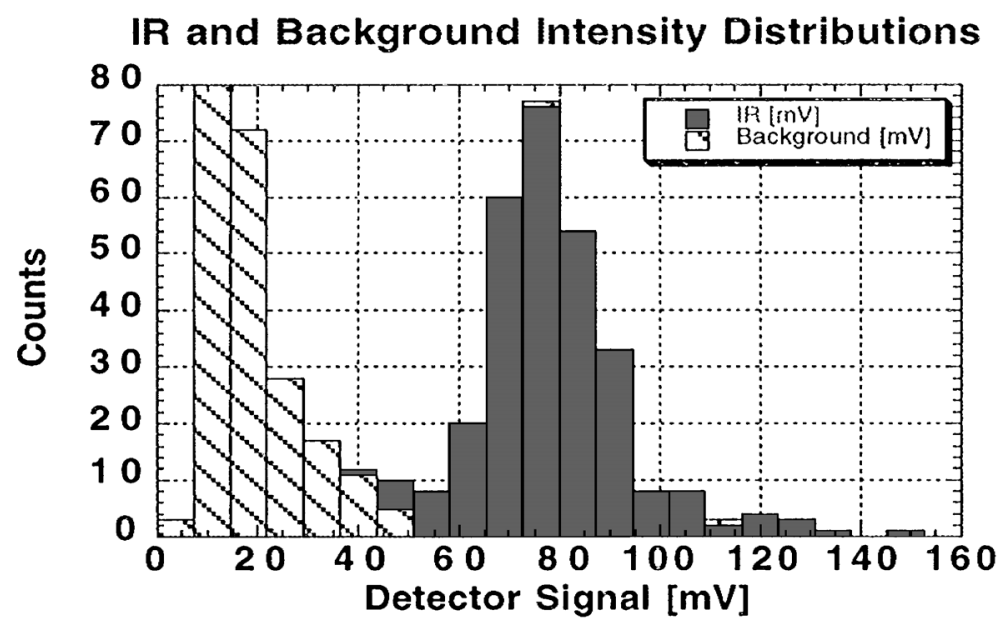

Fig. 28. Intensity distribution of the IR and background signals for a mean charge $Q=$ $0.56 \mathrm{nC}$, standard deviation of $0.007 \mathrm{nC}$, IR mean $=78 \mathrm{mV}$, standard deviation $=14.3 \mathrm{mV}$; background mean $=18.7 \mathrm{mV}$, standard deviation $=9.1 \mathrm{mV}$.

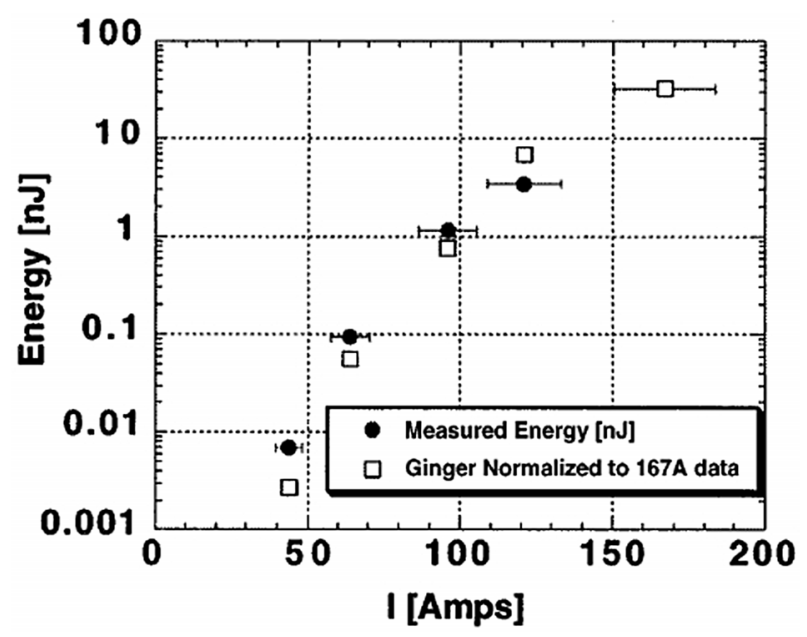

Fig. 29. Measured average FEL output energy (nJ) compared to Gingersimulations for different electron beam peak currents. From reference [Hogan 1998b].

A much larger gain, $3 \times 10^{5}$ at $12 \mu \mathrm{m}$, was obtained in the same year by a UCLAKurchatov Institute-LANL-SLAC collaboration, using a 2-m-long undulator [Hogan 1998b] and an existing Los Alamos National Laboratory (LANL) linac. The results are shown in Figures 29 and 30. This was an important and timely experiment. As we will see later it played a critical role in obtaining the initial financial support for LCLS. It demonstrated the capability of a single pass, high gain SASE-FEL, and its advantages over other short wavelength electromagnetic radiation sources. Saldin and his group [Saldin 1999] independently analyzed the experimental data from this 9 experiment, fully confirming the agreement between the experiment, the theory and the simulations. 


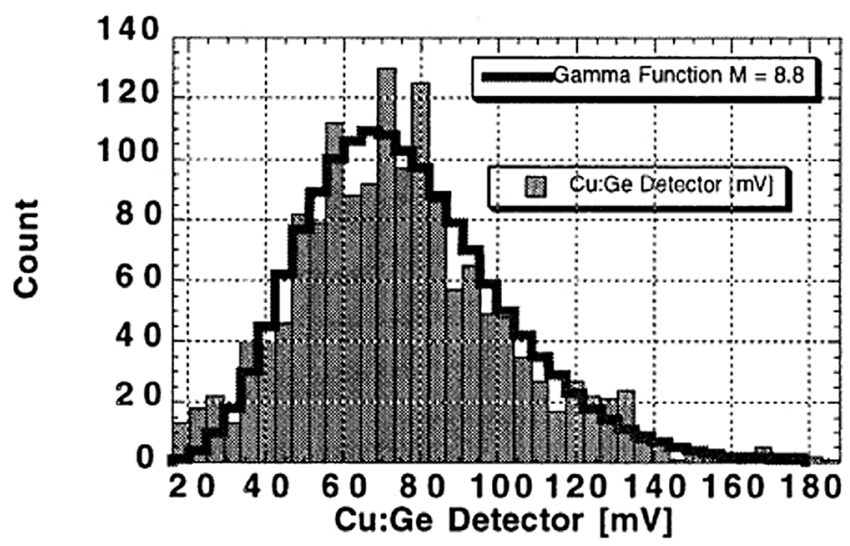

Fig. 30. Measured output intensity fluctuations for individual $2 \mathrm{nC}$ micropulses compared to the predicted gamma distribution function. From reference [Hogan 1998b].

Other theoretical predictions, like the characteristic electron beam microbunching induced by the FEL collective instability, were also measured directly in this experiment [Tremaine 1998] observing coherent transition radiation. One other experiment, at infrared wavelength, by a Los Alamos group, followed shortly after [Nguyen 1998].

The analysis of these experiments, and other experiments to follow, was much helped by the developments of numerical simulation codes which included all the known FEL physics and also a realistic representation of the electron beam properties and its six-dimensional phase space distribution. Particularly important were the codes Ginger, developed by Fawley [Fawley 2001], and Genesis, developed by Reiche [Reiche 1999]. By allowing the evaluation of the effect of a realistic electron beam, of diffraction and of temporal spiking effects, these codes have been instrumental also in the design of LCLS and other short wavelength FELs.

After these experiments the only missing part for a full test of the theory was reaching saturation, that was expected to occur around a gain thirty times larger than that obtained in the UCLA-Kurchatov-LANL-SLAC experiment.

In 2000 and 2001, three SASE-FELs reached saturation with gain larger than 107 : LEUTL, at Argonne, at 530 and $320 \mathrm{~nm}$ in a 20-m-long undulator [Milton 2000; 2001; Andruszkow 2000]; VISA, a BNL-SLAC-LLNL-UCLA collaboration, in a 4-mlong undulator at $800 \mathrm{~nm}$ [Tremaine 2001; Murokh 2003]; and TTF at DESY, at $92 \mathrm{~nm}$, the shortest wavelength at that time for an FEL, with a 15-m long undulator [Ayvazyan 2002]. All the data obtained in these experiments agree well with the theoretical predictions on exponential growth, intensity fluctuations, saturation, and dependence on electron beam parameters. The intensity and gain measurements for these experiments are shown in Figures 31-33. These results, at different wavelengths, from the visible to the UV, strongly support the validity of the FEL SASE theory and the feasibility of an X-ray FEL. They also provide very useful and important experience for the design and construction of short wavelength FELs.

Another line of development for short wavelengths FELs, based on the use of harmonics, was also being studied. The micro bunching produced in an FEL is rich in harmonics, making possible to generate and amplify harmonics of the fundamental radiation wavelength [Boscolo 1982; Murphy 1985; Huang 2000]. Harmonics amplification in SASE FELs was measured following the first high gain experiment [Tremaine 2002a; 2002b]. 


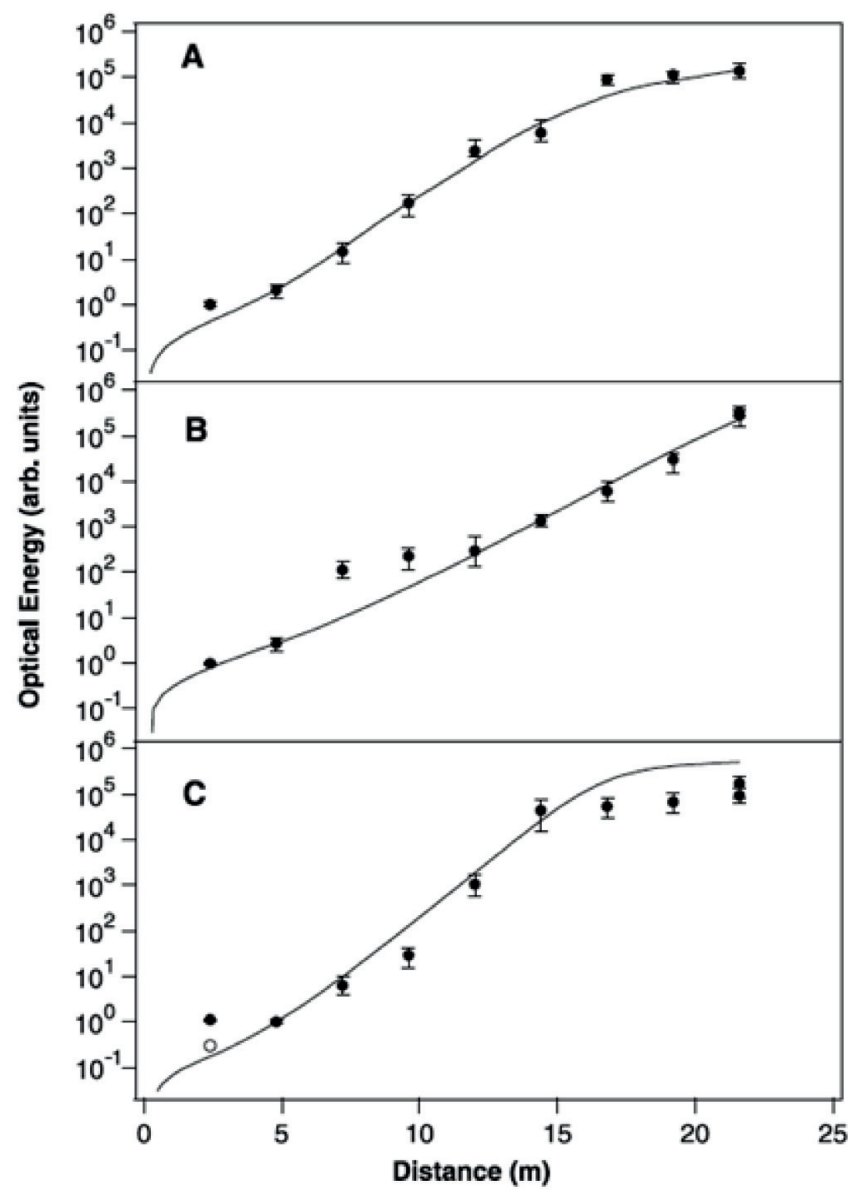

Fig. 31. Intensity as a function of distance along the undulator, under various electron beam conditions. (A) 530-nm saturated conditions. (B) 530-nm unsaturated conditions. (C) 385-nm saturated conditions. The solid curves represent GINGER simulation results. From reference [Milton 2001].

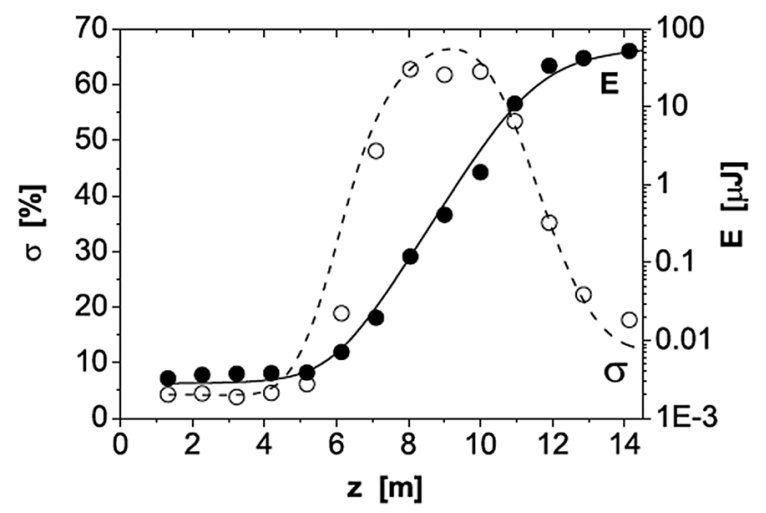

Fig. 32. Average radiation pulse energy (solid circles) and rms energy fluctuations in the radiation pulse (empty circles) as a function of the active undulator length. The wavelength is $98 \mathrm{~nm}$. Circles: experimental results. Curves: numerical simulations. From reference [Ayvazyan 2002]. 


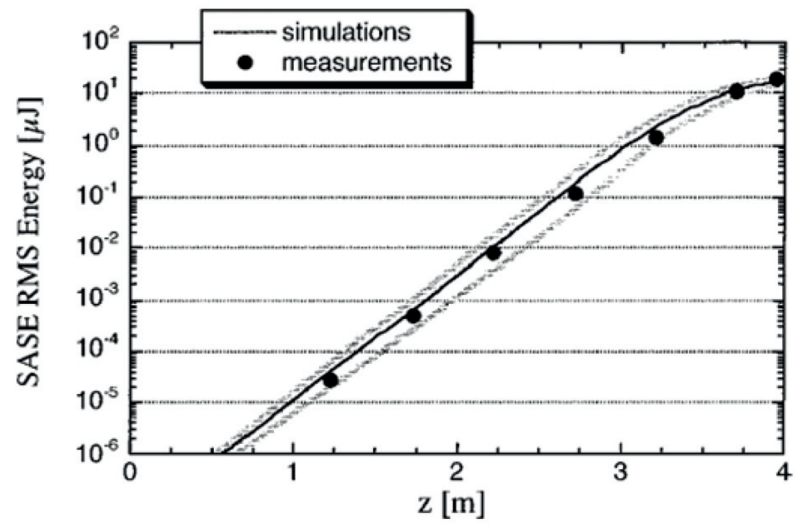

Fig. 33. Measured SASE intensity evolution along the undulator length and numerical simulations (gray lines are the rms boundaries of the set of GENESIS runs). The amplification curve yields a power gain length of $17.9 \mathrm{~cm}$ and saturates near the undulator exit. From reference [Murokh 2003].

The possibility of using harmonics to operate the FEL at short wavelengths using a cascade of undulators tuned at the fundamental and its harmonics, and an input seed laser signal to modulate the beam energy at a wavelength which is a multiple of the final one, was considered by Bonifacio and his group [Bonifacio 1990]. The beam energy modulation is transformed into a longitudinal density modulation, taking advantage of the difference in path length, in a magnetic field, for electrons of different energies. Yu and collaborators [Yu 1991; 2000a] developed another method, High Harmonics High Gain (HGHG) FELs, of using harmonics, sub-harmonics seeding and high gain for short wavelength FEL. This approach was developed and tested initially at Brookhaven [Ben Zvi 1991; Yu 2000b; 2003], and was later considered by many other groups for the design of seeded soft X-ray FELs. This concept is used in the design of the Fermi@Elettra soft X-ray FEL [Allaria 2006], now in operation at the Trieste Synchrotron Laboratory.

These systems do not relax the electron beam phase-space density requirements needed to reach a given wavelength, but offer the advantage of a reduced line-width and the absence of spiking, yielding an improved longitudinal coherence, in some cases approaching a few times the transform limit. However, their realization becomes more complicated if the FEL wavelength is a large harmonic of the input signal used to start the process, and might be problematic at wavelength of about $1 \mathrm{~nm}$ or shorter.

\section{LCLS: The first hard X-ray FELs}

The years 1998-1999 were very important for X-ray FEL development. The Los Alamos-UCLA-Kurchatov-SSRL experiment, the first to demonstrate large gain [Hogan 1998] for a SASE amplifier, gave much needed support to the LCLS project. The LCLS design study group, led by Max Cornacchia, completed and published the LCLS Conceptual Design Report [Cornacchia 1998] in December 1998. The report addressed all the important issues about the feasibility of a 1.5 to $15 \AA$ FEL. It proposed a 3 years construction schedule starting in 2001, with focused R\&D during 1999 and 2000 .

The Basic Energy Science division of the US Department of Energy (DOE) formed a new panel, chaired by Stephen Leone, to consider again the question of the development and construction of coherent light sources, following the previous Birgenau 6 18 19

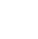

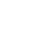

5

6

7

8

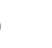

.

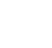

.
5 
report. The charge to the panel was given in a letter to Leone in September 1998: "we would like your panel to address two primary questions:

(1) What new science will be enabled by novel coherent light sources? Besides the obvious increases in brightness there are fundamental differences in peak intensity and coherence properties between the second and third generation synchrotron sources and the envisioned new light sources. How can the high intensities, coherence, and temporal properties of novel sources be utilized to provide new probes of matter using photons? In what fields will the new light sources have the most significant impact?

(2) Given the present state of science and technology, what might be a reasonable research and development plan for novel coherent light sources in the next five years? How would such sources be configured (individual laboratories, modest user centers, large-scale facilities, etc.) and how might they serve the potential user community?"

The panel organized a workshop at Gaithersburg, Maryland, in January 1999. Cornacchia presented the work of the Design Study Group and Pellegrini presented the results of the large gain Los Alamos-UCLA-Kurchatov-SSRL experiment. These elements played an important role in the deliberation of the Panel [Leone 1999], which recommended, for the first time, to DOE to support the development of X-ray FELs:

“... DOE should pursue the development of coherent light source technology in the hard X-ray region as a priority. This technology will most likely take the form of a linac-based free electron laser device using self-amplified stimulated emission or some form of seeded stimulated emission...

... Provisional support should be provided for a highly focused and fiscally responsible set of investigations to determine the feasibility and design of a $1.5 \AA$ coherent light source, ...

... LANL (together with UCLA, SSRL, and the RRC-Kurchatov Institute) has been a major player in proving SASE at the 12 micron level, which was crucial to the X-FEL development."

Another important element for the positive recommendation was the report, "The First Experiments" [Gopal 2003], by a group led by Gopal Shenoy and Joachim Stohr, showing the great scientific interest and unique capabilities of LCLS.

DOE accepted the Leone's Panel recommendation and provided initial funding for R\&D and to prepare a Conceptual Design Report by 2001. This was a very important moment in the history of LCLS and in general of X-ray FELs. Argonne National Laboratory joined the collaboration In December 1998 and Brookhaven National Laboratory joined in April 1999. The initial funding was divided between the six institutions now forming the LCLS collaboration: SLAC, \$800000, UCLA \$214000, Livermore $\$ 185000$, Argonne $\$ 165000$, Brookhaven $\$ 75000$, Los Alamos $\$ 60000$. The distribution between the several R\&D areas is shown in Figure 34. Part of the funding for SLAC, UCLA and Brookhaven was used for the preparation of the VISA SASE FEL experiment, discussed before, that reached saturation in 2001. Livermore received support for the development of X-ray optics, Argonne for the undulator magnet. The largest amount was dedicated to the photoinjector work, as always recognized to be a critical element for the success of the project.

A more formal project management structure was created, with Cornacchia as the project leader reporting to Keith Hodgson, the chair of the Synchrotron Radiation Laboratory at SLAC. A Scientific Advisory Committee for LCLS, with Joachim Stohr and Gopal Shenoy co-chairs, was also created. In addition to overseeing the project development and the FEL R\&D, another very important task was the preparation of the LCLS scientific program. The Scientific Advisory Committee received 


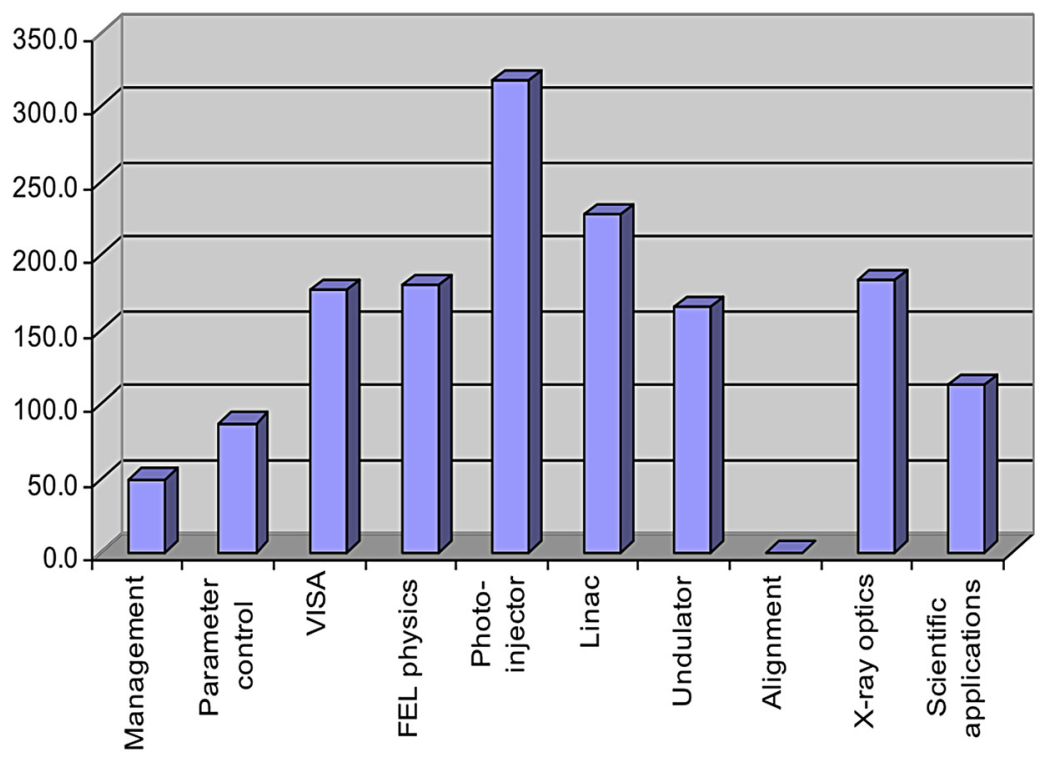

Fig. 34. The distribution of R\&D funds, as presented by Cornacchia to the SLAC Scientific Policy Committee in April 1999, based on the funding proposed by the Leone's Panel.

experimental proposals using the LCLS novel properties, such as the coherence, large peak power, short sub-picosecond pulses, by several groups of X-ray scientists. The proposals are described in the report "LCLS, the first experiments" [Gopal 2003]. At the end six major areas of research, called the six experiments, were identified, and presented to a DOE Basic Energy Science Advisory Committee in October 2000.

The main areas identified for initial research are: Atomic Physics Experiments; Plasma and Warm Dense Matter Studies; Structural Studies on Single Particles and Biomolecules; Femtochemistry; Studies of Nanoscale Dynamics in Condensed Matter Physics; FEL Physics and Development.

It was also clear that the LCLS was quite different from storage ring based synchrotron radiation sources, and that it would be important to move ahead to X-rays pulses shorter than the design $230 \mathrm{fs}$, and also to control the pulse intensity and duration to optimize each experiment. Given the strong connection between the X-ray laser characteristics and the experimental program another workshop, "Physics of, and Science with X-ray FELs", was organized at Arcidosso, in Ialy in September, 2000, bringing together the scientists working on the generation of coherent X-rays with those preparing to use them in the six experimental areas just mentioned [Chattophadyay 2001].

After giving a very important contribution to the X-ray laser development, as one of the leaders of the VISA experiment and leader of the Design Study group, Cornacchia left LCLS in the middle of 1999 and retired from SLAC a few years later. Ewan Paterson took over the position while a search for a new project leader was ongoing. The position was given to John Galayda, who had already successfully lead the construction of the Advanced Photon Source at Argonne, and moved to SLAC to be the LCLS project manager in April 2001.

A new LCLS Conceptual Design Report was completed in 2002 [LCLS 2002]. By this time, the three SASE-FEL experiments mentioned before, [Milton 2000; Andruszkow 2000; Tremaine 2001], had reached saturation giving theoretical and experimental support and other useful information for the LCLS development. 


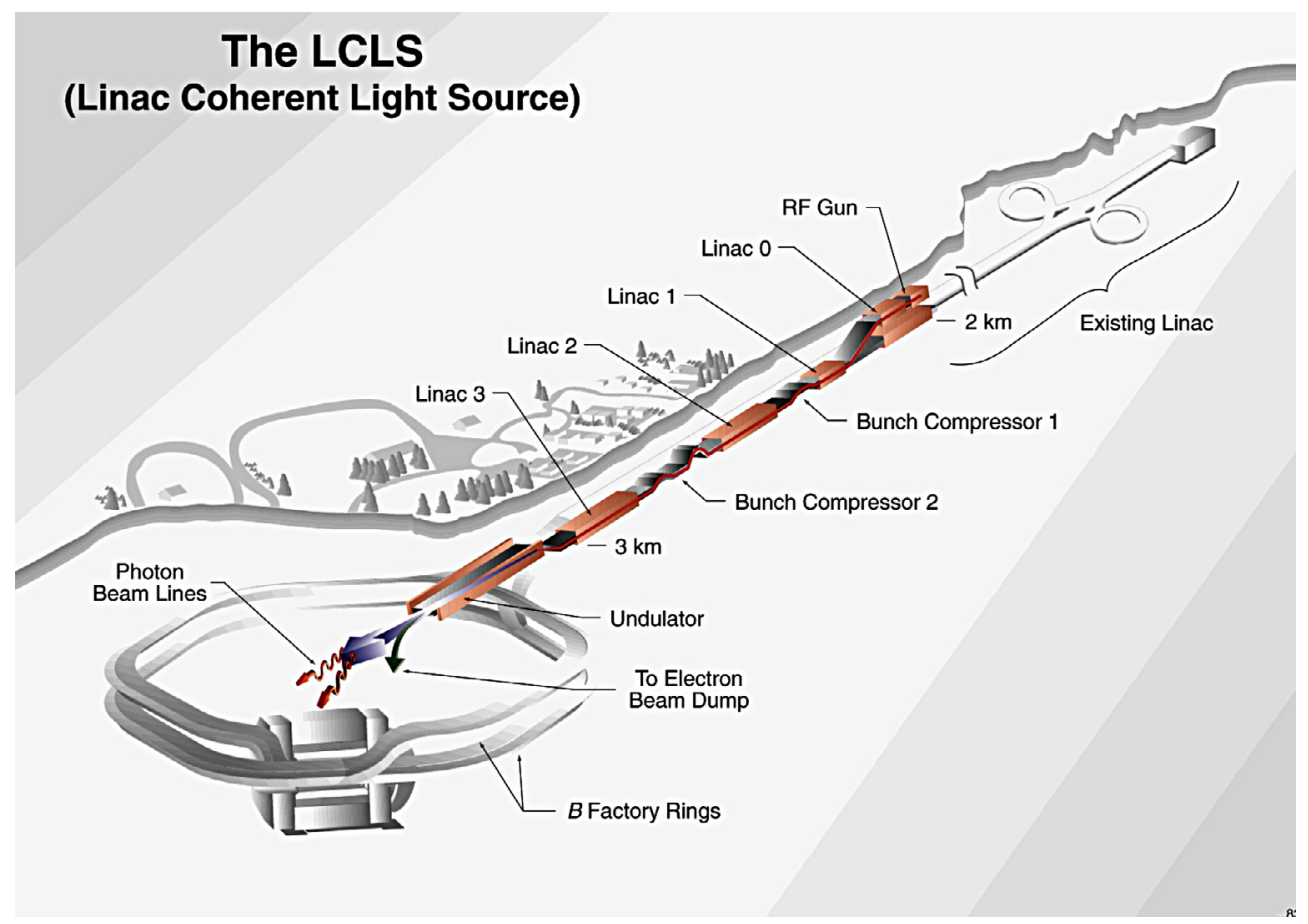

Fig. 35. LCLS schematic. The last $\mathrm{km}$ of the SLAC linac is used to accelerate the beam from the photo-injector to about $14 \mathrm{GeV}$, and compress the bunch to a length of about $30 \mu \mathrm{m}$ or shorter.

Following the presentation of the Conceptual Design Report, DOE provided funding for engineering development. The project was well on its way to the construction stage and to completion and first lasing at $1.5 \AA$ by the spring of 2009 .

During the LCLS engineering and construction period, the Tesla Test Facility FEL, now called FLASH, the soft X-ray FEL at DESY, increased the beam energy to about $1 \mathrm{GeV}$ and decreased the lasing wavelength, finally reaching $6.5 \mathrm{~nm}$ in 2007 [Faatz 2009]. Experiments at FLASH started in 2005, and have since generated many exciting results. One example is the coherent diffraction imaging experiment [Chapman 2006], using a $32 \mathrm{~nm}, 25 \mathrm{fs}, 20 \mu \mathrm{J}$ pulse without damaging the sample, opening the way to the more recent experiments on nano-crystals and viruses at SLAC.

The construction of the LCLS accelerator-undulator system and part of the X-ray experimental facilities and diagnostics were completed in the spring of 2009. Two other national laboratories, Argonne and Lawrence Livermore, participated in the project. Argonne was given the responsibility for the undulator construction. Livermore was responsible for the X-ray instrumentation and diagnostics following the undulator. SLAC, on whose campus LCLS is located, had the responsibility for the overall design, construction and integration of the system, including the instrumentation and facility for experiments using the X-ray photons. John Galayda has been the project manager during the final design, construction and commissioning effort, from 2001 to 2009.

A schematic view of the LCLS on the SLAC campus is shown in Figure 35 and the main electron beam, undulator and X-ray pulse characteristics are given in Table 4 . The electron source is a photo-injector, an improved version of the BNL/SLAC/UCLA design [Palmer 1997], producing a beam with a record breaking normalized emittance, $0.4 \times 10^{-6} \mathrm{~m}$ at a charge of $0.4 \mathrm{nC}$, and as low as $0.15 \times 10^{-6} \mathrm{~m}$ at $0.02 \mathrm{nC}$. The original 
Table 4. LCLS initial experimental characteristics.

\begin{tabular}{lcc}
\hline Electron beam & & \\
\hline Energy, Gev & 13.6 & 13.6 \\
Bunch charge, pC & 250 & 20 \\
Normalized emittance, mm mrad & 0.5 & 0.14 \\
Peak current, kA & 3 & 3 \\
Relative energy spread, \% & 0.01 & 0.01 \\
\hline Undulator & & \\
\hline Period, cm & 3 & 3 \\
Undulator parameter & 3.5 & 3.5 \\
Length, m & 130 & 130 \\
\hline X-ray characteristics & & \\
\hline Wavelength, nm & 0.15 & 0.15 \\
Peak Power, GW & 20 & 25 \\
Gain length, m & 3.3 & 3.3 \\
Pulse length, rms, fs & 60 & $<10$ \\
Line width, \% & $5 \times 10^{-4}$ & $2 \times 10^{-4}$ \\
\hline
\end{tabular}

design goals were a transverse emittance of about $1 \mathrm{~mm}$ mrad at a charge of $1 \mathrm{nC}$. Research and development work done at the Gun Test Facility at SLAC [Schmerge 1999], pointed to some relevant technical improvements that were added to the design. David Dowell, who had joined the LCLS group in 2001, led the final injector design and construction, including the gun and the initial linac sections accelerating the beam to an energy of about $150 \mathrm{MeV}$ with a peak current of $100 \mathrm{~A}$ at $1 \mathrm{nC}$ charge. The injector was optimized for space charge effect reduction and to provide the emittance needed to fully satisfy the LCLS requirements [Alley 1999; Ferrario 2000].

After the injector the electron bunch is compressed twice during the acceleration to reach a peak current of $3 \mathrm{kA}$, while preserving the transverse phase-space and keeping the electron relative energy spread at a value of $10^{-4}$ or better. The bunch compressors consist of a series of magnetic chicanes, producing an electron path length depending on the electron energy. The electron energy distribution is made a linear function of its longitudinal position in the accelerator radio frequency system. When the beam is run through the magnetic chicanes the head particle is delayed respect to the tail particle, reducing the bunch length. The system has been designed and located so that the nonlinearities in the compression and acceleration process (due to longitudinal wakefields, radio-frequency voltage curvature, and second order momentum compaction) are approximately compensated. A small X-band radio-frequency cavity is also used prior to the first compressor to linearize and stabilize the system [see LCLS 2002, p. 7-1 and following].

Even if the initial design called for a $1 \mathrm{nC}$ charge, the injector was designed with the flexibility to operate at lower charges [see LCLS 2002, p. 7-1 and following], making good use of progress in the radio frequency control system. The study of the emittance and current scaling with the electron bunch charge [see LCLS 2000, p. 6-3], and the development of advanced simulation techniques to follow the electron beam during the generation, acceleration, compression process, and the radiation pulse in the FEL process, gave the possibility of examining in detail the optimum choice of the electron bunch charge for LCLS [Borland 2002]. In fact a detailed analysis, based on the scaling laws and a consideration of collective, high intensity, effects in the gun and during acceleration and bunch compression, led to the conclusion that a smaller charge, 250 pC, was a better choice [Reiche 2002].

Collective, high intensity effects can degrade the beam quality, and much care has been taken in the LCLS design to minimize the damage, including dividing the 
bunch compression in two stages at different beam energies, as suggested initially by Sessler and developed by Tor Raubenheimer. One particular effect that received much attention following the LCLS conceptual design report [LCLS 2002] is the coherent synchrotron radiation in the compressor chicanes leading to bunching of the electron beam on the scale of micrometers, the micro-bunching instability [Saldin 2002; Heifets 2002; Huang 2002; Stupakov 2003]. It was also pointed out that the longitudinal space charge fields [Saldin 2003] are an additional effect driving the micro-bunching instability. To control this effect a new element, the laser heater, was introduced in LCLS design [Galyda 2003; Huang 2004]. The laser heater uses the resonant interaction between a laser field and electrons propagating in an undulator to modulate the electron energy and thus effectively increase the electron beam energy spread. The larger spread induces a Landau damping of the instability, thus reducing the growth of micro-bunching. This new elements has been introduced at the end of the injector section of LCLS, before the first bunch compressor. It successfully helped to control the micro-bunching instability and improve the FEL performance [Huang 2010].

After reaching its final energy at the linac exit, the LCLS electron beam enters a $130 \mathrm{~m}$ long permanent magnet undulator, divided in $3.4 \mathrm{~m}$ long segments, separated by sections where electron beam position monitors (BPMs) and quadrupole focusing magnets are installed [LCLS 2002]. The undulator magnetic field must satisfy stringent requirements, a relative error tolerance of about 1 part in ten thousand or better. Since the electron beam and the X-ray beam must overlap over a distance at least equal to one gain length, about $10 \mathrm{~m}$, to avoid a reduction of the FEL gain, the electron beam alignment along the undulator axis must be kept within a few microns [Gluskin 2001; Nuhn 2009].

This level of alignment precision is not easily achievable with existing mechanical survey methods. For this reason, a beam-based alignment technique has been developed and used at LCLS [Emma 1999]. It uses measurements of the beam position, from monitors located between the undulator modules, to observe the effect on the electron bunch location of a large change of the electron beam energy. For an ideal alignment the beam position does not change with energy. The observed changes, due to alignment errors, are analyzed to determine the best trajectory corrections, applied using correcting magnets. The procedure can be repeated to converge to an alignment within the required tolerances.

Another innovation was introduced in the undulator design [Vasserman 2004], to facilitate reaching the magnetic field tolerances. The ideal electron trajectory in the undulator is in the horizontal plane. The geometry of the upper and lower undulator pole faces was changed from parallel to canted respect to each other, with a canting angle of about $5.5 \mathrm{mrad}$. In this geometry the magnetic field and undulator parameter depend on the electron beam's horizontal position. In addition each undulator module can be moved horizontally with high precision, giving the possibility of matching the undulator parameter to the required tolerance.

An advantage of this innovative solution is that, as the electrons lose energy to the X-ray beam, the magnetic field can be reduced to compensate the energy change and keep the resonant wavelength constant, keeping the electron oscillations and the electromagnetic wave in phase. This idea was first proposed and studied by Kroll et al. [Kroll 1981], to increase the energy transfer from the electron beam to the radiation field. It was demonstrated in the Livermore experiment [Orzechowski 1985] discussed before. William Fawley et al. [Fawley 2002] studied tapering the undulator magnetic field for LCLS to increase the output power. This possibility has been verified experimentally during initial operation of LCLS [Ratner 2009].

The X-ray pulse at the undulator exit has a radius of about $10 \mu \mathrm{m}$, and an angular divergence of $0.5 \mu$ radians. The electron beam position and pointing error at 
the undulator entrance must match these values. This is achieved controlling the trajectory between the linac and the undulator entrance, using correcting magnets.

The combination of all the requirements on the electron beam 6-dimensional phase space, its trajectory, and undulator alignment and field quality control represents a formidable challenge for accelerator physics and technology, a challenge that the LCLS group has met with great success. Initial lasing at $1.5 \AA$ was obtained as soon as the beam was sent in the undulator, a remarkable success. Practically all other FELs preceding LCLS had required a much longer and difficult commissioning period.

After the initial results at $1.5 \AA$ [1], new extreme pulse compression schemes with reduced bunch charge have been demonstrated at the LCLS, with high-power X-ray pulse durations of $<10$ fs [Ding 2009a; Ding 2009b]. Both results are reported in Table 4.

Since April 2009 the LCLS has been operating successfully in the full wavelength design range, 15 to $1.5 \AA$. Initial experiments in the area of atomic and molecular physics have already been performed. It is a testimony to the outstanding work done by the design and construction group that even during this initial period of operation the X-ray beam has beam made available to the experimental groups more than $92 \%$ of the scheduled time As Paul Emma recently said: "This encouraging success story demonstrates the real practicality and the great potential of future FELs, which are now well grounded as stable and reliable light sources."

\section{Conclusions and future directions}

The success of LCLS opens the door to many new developments, as discussed and reviewed recently [Pellegrini 2011]. One is production of coherent photons of higher energies, up to $50 \mathrm{keV}$ or more. Another is production of single spike, fully longitudinally coherent, short pulses, shorter than 1 fs, at very low electron bunch charge, around a few pC [Rosenzweig 2008; Reiche 2008], or the reduction of the line width to a value near the transform limit by self-seeding in a two undulators plus monochromator system [Feldhaus 1997], or seeding with an external laser and lasing on higher harmonics. The option of an X-ray FEL oscillator [Lindberg 2009] has been proposed and studied recently to obtain X-ray pulses with extremely small line-width, about $10^{-6}$ to $10^{-7}$.

The European XFEL, under development at DESY and scheduled for end of construction in 2015, will increase the average power and brightness over LCLS. XFEL accomplishes this increase by using superconducting linac to augment the bunch repetition rate from $120 \mathrm{Hertz}$ to $27 \mathrm{kHz}$.

The next generation of Soft X-ray FELs, following FLASH, will cover the nanometer wavelength range with variable polarization radiation, and repetition rates of $100 \mathrm{~Hz}$, like the Fermi [De Ninno 2009] soft X-ray FEL under construction at Trieste, or in the $0.1-3 \mathrm{MHz}$ region for superconducting linac FELs being studied in the UK [Walker 2008] and Lawrence Berkeley National Laboratory [Belkacem 2007].

Considering their unique properties, FELs will play a very important role in the future of atomic and molecular science. The last step in their development will be a reduction in size and cost to make them available to a larger community of scientists. Work in this direction is being carried out right now at several laboratories and universities, to develop high frequency linacs and laser-plasma based electron accelerators, to reduce the accelerator length by one to three orders of magnitudes. New ideas in electron sources and undulators, reducing the period from centimeters to millimeters, will also help to develop future low cost, compact FELs, while keeping unchanged the X-ray pulse characteristics. 
Acknowledgements. I wish to express my gratitude to Hermann Winick, Max Cornacchia, James Rosenzweig, Alexander Varfolomeev, James Murphy, John Galayda, Andy Sessler for reading the manuscript and sharing with me their time and knowledge over many years. I also want to thank all the other scientists, post-doctoral fellows and students, too many to quote, that helped me with discussions and information.

\section{References}

Allaria, E. et al. 2006. FERMI@ELETTRA: a seeded FEL facility for EUV and soft X-rays. Proc. of the 2006 International Free Electron Laser Conf., Berlin, pp. 166-169

Alley, R. et al. 1999. The design for the LCLS RF Photoinjector. Nucl. Instr. Meth. A 429: 324-331

Andruszkow, J. et al. 2000. First observation of self-amplified spontaneous emission in a free-electron laser at $109 \mathrm{~nm}$ wavelength. Phys. Rev. Lett. 85: 3825-3829

Arthur, J., G. Materlik and H. Winick (Eds.) 1994. Workshop on Scientific Applications of Coherent X-Rays. SLAC Rep., p. 437

Ayvazyan, V. et al. 2002. Generation of GW radiation pulses from a VUV free-electron laser operating in the femtosecond regime. Phys. Rev. Lett. 88: 104802

Babzien, M. et al. 1998. Observation of self-amplified spontaneous emission in the nearinfrared and visible wavelengths. Phys. Rev. E 57: 6093-6096

Bane, K. 1987. Wakefield effects in a linear collider. Amer. Inst. Phys. Conf. Proc. 153: 971-981

Batchelor, K., H. Kirk, K. McDonald, J. Sheehan and M. Woodle. 1988. Development of a High Brightness Electrpon Gun for the Accelerator Test Facil;ity at Brookhaven National Laboratory. Proc. of the 1988 European Particle Accelerator Conf. Rome, pp. 54-958

Becker, W. and J.K. McIver. 1983. Fully quantized many-particle theory of a free-electron laser. Phys. Rev. A 27: 1030-1043

Becker, W. and M.S. Zubairy. 1982. Photon statistics of a free-electron laser. Phys. Rev. A 25: $2200-2207$

Belkacem, A. et al. 2007. Design studies for a high repetition rate FEL facility at LBNL, Synchrotron Radiat. News 20: 20-27

Ben-Zvi, I., L.F. Di Mauro, S. Krinsky, M.G. White and L.H. Yu. 1991. Proposed UV FEL user facility at BNL. Nucl. Instr. Meth. A 304: 181-186

Bertolotti, M. 2005. History of the laser. Institute of Physics Publishing, Bristol

Birgenau, R.J. et al. 1997. Report of the Basic Energy Sciences Advisory Committee Panel on D.O.E. synchrotron radiation sources and science, http://www.aps.anl.gov/Science/ Reports/1997/besac.pdf

Bonifacio, R., F. Casagrande and G. Casati. 1982. Cooperative and Chaotic Transition of a Free Electron Laser Hamiltonian Model. Opt. Commun. 40: 219-223

Bonifacio, R., F. Casagrande and C. Pellegrini. 1987. Hamiltonian model of a free-electron laser. Opt. Commun. 61: 55-60

Bonifacio, R., L. De Salvo Souza, P. Pierini and E.T. Scharlemann. 1990. Generation of XUV light by resonant frequency tripling in a two-wiggler FEL amplifier. Nucl. Instr. Meth. A 296: 787-790

Bonifacio, R., L. De Salvo, P. Pierini, N. Piovella and C. Pellegrini. 1994. Spectrum, Temporal Structure and Fluctuations in a High-Gain free-electron laser starting from noise. Phys. Rev. Lett. 73: 70-73

Bonifacio, R., C. Pellegrini and L. Narducci. 1984. Collective instabilities and high-gain regime in a free electron laser. Opt. Commun. 50: 373-378

Borland, M. et al. 2002. Start-to-end simulation of self amplified spontaneous emission freeelectron lasers from the gun through the undulator. Nucl. Instr. Meth. A 483: 268-272

Bosco, P., W.B. Colson and R.A. Freeman. 1983. Quantum/classical mode evolution in free electron laser oscillators. IEEE J. Quantum Electron. QE-19: 272-281

Boscolo, I. and V. Stagno. 1982. A Study of a transverse optical klystrin in Adone (TOKA). Nucl. Instr. Meth. A 198: 483-496 
Carlsten, B.E. 1989. New photoelectron injector design for the Los Alamos national laboratory XUV FEL accelerator. Nucl. Instr. Meth. A 285: 313-319

Chapman, H. et al. 2006. Femtosecond diffractive imaging with a soft-X-ray free-electron laser. Nature Phys. 2: 839-843

Chapman, H. et al. 2011. Femtosecond X-ray protein nanocrystallography. Nature 470: 73-78

Chattopadhyay, S., M. Cornacchia, C. Pellegrini and I. Lindau (Eds.) 2001. Physics of, and Science with, X-ray free-electron lasers. American Instit. of Physics Conf. Proc. 581: $1-236$

Colson, W.B. 1977. One-body electrodynamics in a free electron laser. Phys. Lett. A 64: 190-192

Cornacchia, M. and H. Winick (Eds.) 1992. Proc. of a Workshop on IV Generation Light Sources, SSRL/SLAC Rep. 92/02

Cornacchia, M. et al. 1986. Design concepts of a storage ring for a high power XUV free electron laser. Nucl. Instr. Meth. A 250: 57-63

Cornacchia, M. et al. 1998. LCLS Design Study Report, Stanford Linear Accelerator Center, SLAC R-521

Csonka, P. 1978a. Suggested method for coherent X-Ray production by combined X-ray and low energy photon pumping. Phys. Rev. A 13: 405-410

Csonka, P. 1978b. Suggestion for X-ray laser holography. Part. Accel. 8: 161-165

Dattoli, G., J.C. Gallardo, A. Renieri, M. Richetta and A. Torre. 1985. Quantum coherence properties of the FEL. Nucl. Instr. Meth. A 237: 93-99

Dattoli, G., A. Marino, A. Renieri and F. Romanelli. 1981. Progress in the Hamiltonian picture of the free-electron laser. IEEE J. Quantum Electron. QE-17: 1371-1387

Deacon, D.A.G. et al. 1977. First operation of a free-electron laser. Phys. Rev. Lett. 38: 892-894

De Ninno, G. et al. 2009. FEL Commissioning of the first stage of Fermi@Elettra. Proceedings of the 2009 FEL Conf., Liverpool, pp. 635-638

Derbenev, Y.S., A.M. Kondratenko and E.L. Saldin. 1982. On the possibility of using a free electron laser for polarization control in a storage ring. Nucl. Instr. Meth. A 193: 415-421

Ding, Y. et al. 2009a. Start-to-End Simulations of the LCLS Accelerator and FEL Performance at Very Low Charge. Proc. of the 2009 Part. Acc. Conf., Vancouver, pp. 2355-2357

Ding, Y. et al. 2009b. Measurements and Simulations of Ultralow Emittance and Ultrashort Electron Beams in the Linac Coherent Light Source. Phys. Rev. Lett. 102: 254801 
Fraser, J.S. and R.L. Sheffield. 1987. High-brightness injectors for RF-driven free-electron lasers. IEEE J. Quantum Electron. QE-23: 1489-1496

Galayda, J. 2003. Private communication

Gallardo, J. 1990. Proceedings of the Workshop Prospects for a $1 \AA$ Free-electron Laser, Sag Harbor, N.Y. Brookhaven National Laboratory Rep. 52273

Gea-Banacloche, J., G.T. Moore and M. Scully. 1984. Prospects for an X-ray free-electron laser. Proc. SPIE 453: 393-401

Gluskin, E. et al. 2001. Optimization of the design for the LCLS undulator line. Nucl. Instr. Meth. A 475: 323-327

Gopal, S. and J. Stohr (Eds.) 2003. LCLS The first experiments SLAC report R-611

Gover, A. and P. Sprangle. 1981. A Unified Theory of Magnetic-Bremsstrahlung, Electrostatic Bremsstrahlung, Compton-Raman Scattering and Cerenkov-Smith Purcell Free Electron Laser. IEEE J. Quantum Electron. QE-17: 1196-1215

Hartemann, S.C. et al. 1994. Initial Measurments on the UCLA RF Photoinjector, Nucl. Instr. Meth. A 340: 219-230

Heifets, S., G. Stupakov and S. Krinsky. 2002. Coherent synchrotron radiation instability in a bunch compressor. Phys. Rev. ST Accel. Beams 5: 064401

Hogan, M. et al. 1998a. Measurements of High Gain and Intensity Fluctuations in a Selfamplified, Spontaneous-Emission Free-electron Laser. Phys. Rev. Lett. 80: 289-292

Hogan, M. et al. 1998b. Measurements of gain larger than $10^{5}$ at $12 \mu \mathrm{m}$ in a self-amplified spontaneous-emission free-electron laser. Phys. Rev. Lett. 81: 4867-4870

Huang, Z. and K.-J. Kim. 2000. Three-dimensional analysis of harmonic generation in highgain free-electron lasers. Phys. Rev. E 62: 7295-7308

Huang, Z. and K.-J. Kim. 2002. Formulas for coherent synchrotron radiation microbunching in a bunch compressor chicane. Phys. Rev. ST Accel. Beams 5: 074401

Huang, Z. et al. 2004. Suppression of microbunching instability in the linac coherent light source. Phys. Rev. ST Accel. Beams 7: 074401

Huang, Z. et al. 2010 Measurements of the linac coherent light source laser heater and its impact on the X-ray free-electron laser performance, Phys. Rev. ST Accel. Beams 13: 020703

Jacobsen, C. and J. Kirz. 1998. X-ray microscopy with synchrotron radiation. Nat. Struct. Biol. (supplement) 5: 650-653

Jerby, E. and A. Gover. 1985. Investigation of the gain regimes and gain parameters of the free electron laser dispersion equation. IEEE J. Quantum Electron. QE-21: 1041-1058

Katsouleas, T.C. et al. 2009. Scientific Assessment of high Power Free-electron Laser Technology. The National Academies Press, Washington D.C.

Kim, K.-J. 1986a. An analysis of self-amplified spontaneous emission. Nucl. Instr. Meth. A 250: 396-403

Kim, K.-J. 1986b. Three-dimensional analysis of coherent amplification and self-amplified spontaneous emission in free-electron lasers. Phys. Rev. Lett. 57: 1871-1874

Kim, K.-J. 1990. Note on RF Photo-Cathode Gun. in Proc. of a Workshop Prospects for a 1 A Free-electron Laser, Sag Harbor, N.Y., Brookhaven National Laboratory Rep. 52273 $122-135$

Kirkpatrick, D.A., G. Bekefi, A.C. Dirienzo, H.P. Freund and A.K. Ganguly. 1989. A high power, $600 \mathrm{~m}$ wavelength free electron laser. Nucl. Instr. Meth. A 285: 43-46

Kondradenko, A.M. and E.L. Saldin. 1980. Generation of coherent radiation by a relativistic electron beam in an undulator. Part. Accel. 10: 207-216

Krinsky, S. and L.H. Yu. 1987. Output Power in guided modes for amplified Spontaneous Emission in a Single Pass Free-electron Laser. Phys. Rev. A 35: 3406-3423

Kroll, N.M. and W.A. McMullin. 1978. Stimulated Emission from relativistic electrons passing through a spatially periodic transverse magnetic field. Phys. Rev. A 17: 300-308

Kroll, N.M., P. Morton and M.N. Rosenbluth. 1981. Free-electron lasers with variable parameter Wigglers, IEEE J. Quantum Elec. QE-17: 1436-1468

LCLS. 2002. LCLS Conceptual Design Report, Stanford Linear Accelerator Center, SLACR-593, http://www.slac.stanford.edu/cgi-wrap/getdoc/slac-r-593.pdf 
Lefevre, A.K., J. Gardelle, G. Marchese, J.L. Rullier and J.T. Donohue. 1999. Self-amplified spontaneous emission and bunching at $3 \mathrm{GHz}$ in a microwave free-electron laser. Phys. Rev. Lett. 82: 323-326

Leone, S. et al. 1999. Report of the DOE Basic Energy Sciences Advisory Committee Panel on Novel Coherent Light Sources. http://www.science.doe.gov/bes/besac/reports.html

Levy, D.H. et al. 1994. Free Electron Lasers and Other Advanced Sources of Light: Scientific Research Opportunities. National Research Council, National Academies Press, http:// www.nap.edu/openbook.php?record_id=9182\&page $=1$

Lindberg, R.R. et al. 2009. Simulation Studies of the X-ray Free-electron Laser Oscillator. Proceedings of the 2009 FEL Conf. Liverpool, pp. 587-590

Liouville, J. 1838. Sur la théorie de la variation des constantes arbitraries. J. Math. Pures Appl. 3: 342-349

Madey, J.M.J. 1971. Stimulated emission of bremsstrahlung in a periodic magnetic field. J. Appl. Phys. 42: 1906-1913

McDonald, K.T. 1988. Design of the laser-driven RF electron gun for the BNL accelerator test facility. IEEE Trans. Electron Devices 35: 2052-2059

Milton, S.V. et al. 2000. Observation of self-amplified spontaneous emission and exponential growth at $530 \mathrm{~nm}$. Phys. Rev. Lett. 85: 988-991

Milton, S.V. et al. 2001. Exponential gain and saturation of a self-amplified spontaneous emission free-electron laser. Science 292: 2037-2040

Moore, G.T. 1984. high-gain small-signal modes of the free-electron laser. Opt. Commun. 52: $46-51$

Moore, G.T. 1985. The high-gain regime of the free electron laser. Nucl. Instr. Meth. A 239: $19-28$

Motz, H. 1951. Applications of the radiation from fast electron beams. J. Appl. Phys. 22: 527-535

Motz, H. 1953. Experiments on radiation by fast electron beams. J. Appl. Phys. 24: 826-833

Murokh, A. et al. 2003. Properties of the ultrashort gain length, self-amplified spontaneous emission free-electron laser in the linear regime and saturation. Phys. Rev. E 67: 066501 (5p)

Murphy, J.B. and C. Pellegrini. 1985a. Generation of high-intensity coherent radiation in the soft-X-ray and vacuum-ultraviolet region, J. Opt. Soc. Amer. B 2: 259-264

Murphy, J.B. and C. Pellegrini. 1985b. Free electron lasers for the XUV spectral region. Nucl. Instrum. And Meth. A 237: 159-167

Murphy, J.B., C. Pellegrini and R. Bonifacio. 1985c. Collective instability of a free electron laser including space charge and harmonics. Opt. Commun. 53: 197-202

Murphy, J.B. and C. Pellegrini. 1990. Introduction to the physics of the free-electron laser. in Laser Handbook, edited by W. Colson, C. Pellegrini and A. Renieri, Elsevier, Amsterdam, pp. 163-219

Neal, R.B. (Ed.). 1967. The Stanford Two Mile Accelerator, W.A. Benjamin Inc., New York. The book has been digitized and can be found at http://www.slac.stanford.edu/library/ 2MileAccelerator/2mile.htm

Nguyen, D.C. et al. 1998. Self-amplified spontaneous emission driven by a high-brightness electron beam, Phys. Rev. Lett. 81: 810-813

Nuhn, H.-D. et al. 2009. LCLS undulator commissioning, alignment, performance. Proceedings of the 2009 FEL Conf., Liverpool, pp. 714-721

Orzechowski, T. et al. 1985. Microwave radiation from a high gain free-electron laser amplifier. Phys. Rev. Lett. 54: 889-892

Palmer, R.V. 1972 Interaction of relativistic particles and free electromagnetic waves in the presence of a static helical magnet. J. Appl. Phys. 43: 3014-3023

Palmer, D.T. et al. 1997. Emittance studies of the BNL/SLAC/UCLA 1.6 Cell Photocathode RF Gun. Proc. of the 1997 Particle Acc. Conf., Vancouver, pp. 2687-2689

Palmer, D.T. 1998. The Next Generation Photoinjector. Stanford University Ph.D. thesis, SLAC Report 500

Pantell, R.H., G. Soncini and H.E. Puthoff. 1968. Stimulated photon-electron scattering. IEEE J. Quantum Electron. QE-4: 905-907 
Pellegrini, C. 1988. Progress Towards a Soft X-ray FEL. Nucl. Instr. Meth. A 272: 364-367

Pellegrini, C. 1990. SASE and Development of an X-Ray FEL. Proc. of the Workshop Prospects for a 1 A Free-electron Laser, Sag Harbor, N.Y. Brookhaven National Laboratory Rep. 52273, pp. 3-12

Pellegrini, C. 1992. A 4 to $0.1 \mathrm{~nm}$ FEL Based on the SLAC Linac. Proc. Workshop IV Generation Light Sources, edited by M. Cornacchia and H. Winick, SSRL/SLAC Rep. 92/02, pp. 364-375

Pellegrini, C. et al. 1993. A 2 to $4 \mathrm{~nm}$ High Power FEL on the SLAC Linac. Nucl. Instr. Meth. A 331: 223-227

Pellegrini, C. et al. 1994. The SLAC Soft X-Ray High Power FEL. Nucl. Instr. Meth. A 341: 326-330

Pellegrini, C. 2001.The Free-Electron Laser Collective Instability and the Development of X-Ray FELs. Proc. of the 2001 Particle Accelerator Conference, IEEE, Chicago, pp. 295299

Pellegrini, C. and S. Reiche. 2004. The development of X-ray free-electron lasers. IEEE J. Sel. Top. Quantum Electron. 10: 1393-1404

Pellegrini, C. 2011. The Challenge of 4th Generation Light Sources. Proc. of the Intern. Part. Acc. Conf., San Sebastian, pp. 3798-3802

Philips, R.M. 1960. The Ubitron, a high-power traveling-wave tube based on a periodic beam interaction in unloaded waveguide. IRE Trans. Electron Devices 7: 231-241

Pierce, J.R. 1962. History of the Microwave-Tube Art. Proc. of the IRE 50, pp. 978-984

Prazeres, R., J.M. Ortega, F. Glotin, D.A. Jaroszynski and O. Marcouillé. 1997. Observation of self-amplified spontataneous emission in a mid-infrared free-electron laser. Phys. Rev. Lett. 78: 2124-2127

Qiu, J., K. Batchelor, I. Ben-Zvi and X.-J. Wang. 1996. Demonstration of emittance compensation through the measurement of the Slice emittance at 10-ps electron Bunch. Phys. Rev. Lett. 76: 3723-3726

Ratner, D. et al. 2009. FEL gain length and taper measurements at LCLS. Proceedings of 2009 FEL Conf., Liverpool, pp. 221-224

Raubenheimer, T.O. 1995. Electron beam acceleration and compression for short wavelength FELs. Nucl. Instr. Meth. A 358: 40-43

Reiche, S. 1999. Genesis 1.3 A Fully 3D Time Dependent FEL Simulation Code. Nucl. Instr. Meth. A 429: 243-248

Reiche, S., C. Pellegrini, J. Rosenzweig, P. Emma and P. Krejcik. 2002. Start-to-end simulation for the LCLS X-ray FEL. Nucl. Instr. Meth. A 483: 70-74

Reiche, S., P. Musumeci, C. Pellegrini and J. Rosenzweig. 2008. Developments of Ultra-Short Pulse Single Coherent Spike for SASE X-Ray FELs. Nucl. Instr. Meth. A 593: 45-48

Robinson, K.W. 1985. Ultra Short Wave Generation. Nucl. Instr. Meth. A 239: 111-118

Roentgen, W.C. 1895. Über eine neue Art von Strahlen. Sitzungsberichtes der Würzburger Physik-medic Gesellschaft

Rosenzweig, J. et al. 2008. Generation of Ultra-short High Brightness Electron Beams for Single Spike SASE FEL Operation. Nucl. Instr. Meth. A 593: 39-44

Rossbach, J. and the TESLA FEL Study Group. 1996. A VUV Free Electron Laser at the TESLA Test Facility at DESY. Nucl. Instr. Meth. A 375: 269-273

Saldin, E.L., E.A. Schneidmiller and M.V. Yurkov. 1998. Statistical Properties of the Radiation from SASE-FEL Operating in the Linear Regime. Nucl. Instr. Meth. A 407: 291-295

Saldin, E.L., E.A. Schneidermiller and M.V. Yurkov. 1999. Numerical simulations of the UCLA/LANL/RRCKI/SLAC experiment on a High Gain SASE FEL. Nucl. Instr. Meth. A 429: 197-201

Saldin, E., E. Schneidmiller and M. Yurkov. 2002. Klystron instability of a relativistic electron beam in a bunch compressor. Nucl. Instr. Meth. A 490: 1-8

Saldin, E., E. Schneidmiller and M. Yurkov. 2003. Longitudinal Space Charge Driven Microbunching Instability in TTF2 linac. report TESLA-FEL-2003-02 Rep., DESY, pp. $1-13$ 
Scharlemann, E.T., A.M. Sessler and J.S. Wurtele. 1985. Optical guiding in a free-electron laser. Phys. Rev. Lett. 54: 1925-1928

Schmerge, J.F. et al. 1999. Photocathode rf gun emittance measurements using variable length laser pulses. Workshop on Free-Electron Laser Challenges II, Harold E. Bennett; David H. Dowell (Eds.), SPIE Conf. Proc. 3614: 22-32

Schneider, J.R. 2010. Photon Science at Accelerator-based Light Sources. Rev. Accel. Sci. Tech. 3: 13-37

Seeman, J. et al. 1991a. Summary of Emttance control in the SLC Linac. Proc. of 1991 U.S. Particle Accelerator Conf., pp. 2064-2067

Seeman, J. et al. 1991b. Multibunch energy and spectrum control in the SLC high energy Linac. Proc. of 1991 U.S. Particle Accelerator Conf., pp. 3210-3213

Seibert, M.M. et al. 2011. Single mimivirus particles intercepted and imaged with an X-ray laser. Nature 470: 78-82

Sprangle, P. and R.A. Smith. 1980. Theory of free-electron lasers. Phys. Rev. A 21: 293-301

Sprangle, P., C.M. Tang and C.W. Roberson. 1985. Collective effects in the free electron Laser. Nucl. Instr. Meth. A 239: 1-18

Stupakov, G. 2003. Theory and observations of microbunching instability in electron machines. Proc. of the 2003 Particle Acc. Conf., Portland, Oregon, pp. 102-106

Tanaka, H. et al. 2011. SACLA Project-Status of beam commissioning. Proc. of 2011 FEL Conf. Shanghai

TESLA. 1995. A VUV free electron laser at the TESLA test facility at DESY. Conceptual Design Report, DESY Print, TESLA-FEL 95-03

Travish, G. et al. 1995. Parametric Studies of an X-ray FEL. Nucl. Instr. Meth. A 358: 60-63

Tremaine, A. et al. 1998. Observation of self-amplified spontaneous-emission-induced electron-beam microbunching using coherent transition radiation. Phys. Rev. Lett. 81: 5816-5819

Tremaine, A. et al. 2001. Saturation measurement of a visible SASE FEL. Proc. of the 2001 Particle Accelerator Conf., Chicago, pp. 2760-2762

Tremaine, A. et al. 2002a. experimental characterization of nonlinear harmonic radiation from a visible self-amplified spontaneous emission free-electron laser at saturation. Phys. Rev. Lett. 88: 204801

Tremaine, A. et al. 2002b. Fundamental and harmonic microbunching in a high-gain selfamplified spontaneous-emission free-electron laser, Phys. Rev. E 66: 03650341

Varfolomee, A.A. et al. 1995. Development of focusing undulators on the basis of Side Magnet Arrays. Nucl. Instr. Meth. A 359: 85-88

Varian, R.H. and S.F. Varian. 1939 A High frequency oscillator and amplifier. J. Appl. Phys. 10: $321-327$

Vasserman, I. et al. 2004. LCLS undulator design development. Proc. of the 2004 FEL Conference, pp. 367-370

Vinko, S.M. et al. 2011. Creation and diagnosis of a solid-density plasma with an X-ray free-electron laser. Nature 482: 59-62

Walker, R.P. 2008. Considerations for a New Light Source for the UK. Proc. of 2008 FEL Conf. Gyeongju, pp. 160-162

Wang, J.-M. and L.-H. Yu. 1986. A transient analysis of a bunched beam free electron Laser. Nucl. Instr. Meth. A 250: 484-489

Weizsäcker, C.F. 1934. Ausstrahlung bei Stössen sehr schneller Elektronen. Z. Phys. 88: $612-625$

Williams, E.J. 1935 Correlation of certain collision problems with radiation theory. $\mathrm{Kgl}$. Danske Videnskab. Selskab Mat.-fys. Medd. 13

Winick, H. et al. 1994. Short wavelength FELs using the SLAC Linac. Nucl. Instr. Meth. A 347: 199-205

Young, L. et al. 2010. Femtosecond electronic response of atoms to ultra-intense X-rays. Nature 466: 46-52

Yu, L.-H., S. Krinsky and R. Gluckstern. 1990. Calculation of Universal Scaling for Freeelectron Laser Gain. Phys. Rev. Lett. 64: 3011-3014 
Yu, L.H. 1991. Generation of intense UV radiation by subharmonically seeded single-pass free-electron lasers. Phys. Rev. A 44: 5178-5193

$\mathrm{Yu}, \mathrm{L} .-\mathrm{H}$. et al. 2000a. First lasing of a high-gain harmonic generation free-electron laser experiment, Nucl. Instr. Meth. A 445: 301-306

Yu, L.-H. et al. 2000b. High-Gain Harmonic-Generation Free-electron Laser. Science 289: 932-934

Yu, L.H. et al. 2003. First Ultraviolet High-Gain Harmonic-Generation Free-Electron Laser. Phys. Rev. Lett. 91: 074801(4p)

Zinth, W., A. Laubereau and W. Kaiser. 2011 The long journey to the laser and its rapid development after 1960. Eur. Phys. J. H 36: 153-181 